\title{
Interval process model and non-random
}

\section{vibration analysis}

(1)

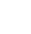

\author{
C. Jiang*, B.Y. Ni, N.Y. Liu, X. Han, J. Liu
}

State Key Laboratory of Advanced Design and Manufacturing for Vehicle Body, College of Mechanical and Vehicle Engineering, Hunan University, Changsha City, P. R.

\section{China 410082}

\begin{abstract}
This paper develops an interval process model for time-varying or dynamic uncertainty analysis when information of the uncertain parameter is inadequate. By using the interval process model to describe a time-varying uncertain parameter, only its upper and
\end{abstract} lower bounds are required at each time point rather than its precise probability distribution, which is quite different from the traditional stochastic process model. A correlation function is defined for quantification of correlation between the uncertain-but-bounded variables at different times, and a matrix-decomposition-based method is presented to transform the original dependent interval process into an independent one for convenience of subsequent uncertainty analysis. More importantly, based on the interval process model, a non-random vibration analysis method is proposed for response computation of structures subjected to time-varying uncertain external excitations or loads. The structural dynamic responses thus can be derived in the form of upper and lower bounds, providing an important guidance for practical safety analysis and reliability design of structures. Finally, two numerical examples 
1 and one engineering application are investigated to demonstrate the feasibility of the interval

2 process model and corresponding non-random vibration analysis method.

3 Keywords: interval process model, time-varying uncertainty, non-random vibration analysis,

4 uncertain dynamic analysis

\section{1. Introduction}

The physical parameters used to describe a structure are often uncertain due to geometrical imperfections, model inaccuracies, or external interferences. Traditionally, these parameters

8 can be identified by random variables which have been well studied in probability theory.

9 Probabilistic approaches [1-3] provide an attractive framework for structural uncertainty analysis, with the premise that a great number of experimental samples are available to construct the precise probability distributions of parameters. However, in practical engineering, one may not be able to build credible probability models for the uncertain parameters due to lack of sufficient data [4]. And researches have indicated that when such information is inaccurate large errors can be incurred in the calculation of the structural failure probability [5].

To deal with the difficulty that sufficient information on the parameters are often not available due to the limitations of test conditions or cost in practical engineering problems, another kind of uncertainty modeling technique, namely the non-probabilistic convex model, has been developed, in which the fluctuation of the imprecise parameters is assumed to fall into a convex set rather than given precise probability distributions. By using the convex model approach, only the variation bounds of the uncertain parameters are required, which in 
engineering practice generally can be identified based on limited samples or just the engineers' experience on the problem. In the early 1990s, Ben-Haim and Elishakoff [5-7] firstly introduced convex models into uncertainty analysis and design of structures. After that, the convex model theory entered a stage of prosperity, and a series of achievements were made in this field. Though different kinds of convex models have been proposed for uncertainty modeling, the interval model and the ellipsoid model presently are the two most widely used ones. Based on the interval model, the anti-optimization approach $[5,8]$ was developed and applied in structural uncertainty analysis and optimization design [9-12] to seek for the least favorable response of a structure under constraints defined by variation bounds of the uncertain variables. The interval perturbation method was proposed to evaluate the range of dynamic responses of structures [13] and to predict the response bounds of an exterior acoustic field with interval parameters [14]. By combining the finite element method (FEM) with interval analysis, the interval finite element method was developed to compute the structural response bounds under parameters' uncertainty [15-17]. Specifically, the concept of interval field was proposed to represent input uncertain parameters and output responses of an FEM analysis [18, 19]. By use of Gram-Schmidt orthogonal transformation, a method of finding the best ellipsoidal convex model fitting the experimental data was put forward [20]. A correlation analysis technique was proposed for uncertainty modeling of the multidimensional ellipsoid model [21]. The ellipsoid model and the interval model were compared for carrying out dynamic response analysis and buckling failure analysis of bars [22]. In recent years, to deal with more complex uncertainty problems several new convex models were also developed such as the super ellipsoid model [23], the multi-ellipsoid model 
$1[24,25]$ and the multidimensional parallelepiped model [26], thus promoting the

2 non-probabilistic convex model theory to a further stage. With the flourishing of convex

3 model theory, some related structural uncertainty analysis and design techniques were also

4 developed, such as non-probabilistic reliability analysis [25, 27-31], probability-interval

5 hybrid reliability analysis [32-34] and uncertain optimization [35, 36], etc.

6 In existing studies, the convex model theory and corresponding analysis methods are 7 mostly developed to solve the time-invariant uncertain problems, where parameters and their

8 uncertainty do not change with time. However, for many practical engineering problems, the

9 uncertainty of structural parameters often has time-varying or dynamic characteristics due to the impact of working environment factors. For time-varying uncertain parameters such as wind excitations, generally the traditional stochastic process model $[37,38]$ can be employed for uncertainty modeling if the precise probabilistic characteristics could be identified. In engineering practice, however, the precise values of these time-varying distribution characteristics are often quite difficult or costly to obtain, since a huge amount of experimental tests are generally required. For example, in design of a bridge structure especially for the long-span bridges, the preliminary explorations on the complicated and changeable local marine environments, such as seawater temperature, waves and tides etc., are essential, while determination of their precise dynamic characteristics for stochastic process modelling generally requires a great number of on-site measurements, which is often costly and time-consuming. Another example is the time-varying surface temperature of a satellite antenna. A fluctuation in the temperature can be caused when the satellite moves along its space orbit around the earth, but the detection on the temperature is often rather 
1 difficult in the distant outer space environment. Hence in the design stage of the satellite

2 antenna, exact probabilistic characteristics of the time-varying surface temperature are often

3 not available. Thus for problems with limited sample data, to develop some new types of

4 quantification models for time-varying uncertainty and corresponding structural uncertainty

5 analysis methods seems very important, which could significantly improve the safety and

6 reliability design level of many practical structures or products.

7 To achieve this goal, this paper firstly proposed a new non-stochastic quantification model

8 for time-varying uncertainty, namely the "interval process model". In this model, only the

9 variation bounds rather than the precise probability distribution are required for a time-varying uncertain parameter, thus greatly reducing its dependency on experimental observations or samples. The formulation of this model is partly based on our recent work, i.e., the convex model process [39]. But quite different from the convex model process, the present model gives a new definition of the important self-correlation function and whereby a new treatment for uncertainty modeling, which thereby makes the subsequent structural uncertainty analysis much more convenient. More importantly, based on the interval process model, a new non-random vibration analysis method is proposed, which aims to provide an effective computational tool for dynamic analysis of many complex structures subjected to uncertain excitations with limited information. The remainder of this paper is organized as follows. Section 2 gives an introduction of the proposed interval process model. Section 3 formulates the non-random vibration analysis method. Numerical examples are investigated in Section 4 before we draw conclusions in Section 5. 


\section{Interval process model}

2

3

4

In traditional stochastic process theory $[37,38]$, a time-varying uncertain parameter $X(t)$ is assumed to be random at arbitrary time, hence it can also be interpreted as a collection of random variables with probability distributions assigned. The correlation between the random variables discretized at two different time points is generally quantified by correlation coefficient or covariance. As stated previously, it generally requires a huge amount of sample data. As shown in Fig.1, for lack of sufficient experimental samples, our interval process model requires only the upper and lower variation bounds of a time-varying uncertain parameter. Compared with the probability information such as the cumulative distribution function (CDF) and the probability density function (PDF), the variation bounds of a parameter are much easier to obtain in practical engineering, which generally can be obtained from a relatively small number of experimental samples or even engineers' experience [40, 41].

In the interval process model, we assume that the variation range of a time-varying uncertain parameter $X(t)$ is enveloped within a pair of upper and lower bounds as shown in Fig. 1. We denote such a time-varying uncertain parameter (or referred to as uncertain process) as $\left\{X^{I}(t), t \in T\right\}$, where $T$ is named as the parameter set or index set, which generally refers to time domain. For arbitrary instant $t_{i} \in T$, the variation range of $X\left(t_{i}\right)$ is an interval,

$$
X^{I}\left(t_{i}\right)=\left[X^{L}\left(t_{i}\right), X^{U}\left(t_{i}\right)\right]
$$

where the subscript $I, L$, and $U$ denote interval, lower bound and upper bound, respectively. We call $X^{I}\left(t_{i}\right)$ as an interval variable, and the characteristics of a conventional interval variable can refer to [42]. Consequently, an interval process $X^{I}(t)$ can also be interpreted as 
1 a "time-varying interval variable". Thus our interval process can be also called

2 "non-stochastic process", "convex model process", “envelope process" or

3 "uncertain-but-bounded process". Based on this, we give the following definitions.

4 Definition 1. A time-varying uncertain parameter $X(t)$ is an interval process if for arbitrary

5 instant $t_{i} \in T, i=1,2, \ldots$, the possible values of $X\left(t_{i}\right)$ can be represented by an interval

$6 X^{I}\left(t_{i}\right)=\left[X^{L}\left(t_{i}\right), X^{U}\left(t_{i}\right)\right]$, where $T$ is a parameter set of $t$.

7 For convenience, we will denote the interval process $\left\{X^{I}(t), t \in T\right\}$ as $X^{I}(t)$ in brief

8 hereafter. And at a certain time $t_{i}$, the interval variable $X^{I}\left(t_{i}\right)$ can also be denoted as $X_{i}$.

9 The sample function of an interval process is denoted as $x(t)$ in this paper. In the interval process model, all possible sample functions of the process should be strictly enveloped 11 within the upper bound function $X^{U}(t)$ and the lower bound function $X^{L}(t)$.

Definition 2. For an interval process $X^{I}(t)$ with the upper bound function $X^{U}(t)$ and the lower bound function $X^{L}(t)$, its middle point function $X^{c}(t)$ and half width function $X^{r}(t)$ are defined as,

Definition 3. The variation coefficient function $X^{V}(t)$ is defined as a ratio of the half width function to the middle point function,

$$
X^{V}(t)=\frac{X^{r}(t)}{X^{c}(t)}
$$

For an interval process $X^{I}(t)$, the middle point function shows its overall variation trend with $t$, and the half width function shows the variation range. The variation coefficient function reflects the degree of uncertainty. In Fig. 2(a) and Fig. 2(b), two cases of the interval 
1 process with constant middle point function and constant half width function are presented. In

2 Fig. 2(c), a case with constant variation coefficient is given.

\section{$3 \quad 2.1$ Classification of the interval process}

$4 \quad$ In many practical problems, the index set $T$ of an uncertain process $\left\{X^{I}(t), t \in T\right\}$ refers

5 to time; in some circumstances, $T$ can be designated as other physical quantities, such as

6 length, temperature and node. Furthermore, when more than a single index is involved, such

7 as the description of the location on a two dimensional plane or in a three dimensional space,

8 a vector $\mathbf{t}$ of the indexes can be utilized to represent the coordinate values. Correspondingly,

9 the non-stochastic field can be established for further uncertainty analysis. In this paper, we only consider interval processes where $t$ is of one dimension, such as time. According to the property of the index set $T$, the interval process $\left\{X^{I}(t), t \in T\right\}$ can be categorized into two classes: 1) if the index set $T$ is a countable set (or discrete in time), this class of process is called discrete interval process, as shown in Fig. 3(a); 2) if the index set $T$ is non-countable (or continuous in time), this class of process is called continuous interval process, as shown in Fig. 3(b).

\subsection{Correlation quantification in interval process} have an influence on that at next time or stage, which is usually referred to as correlation. In this part, our discussion will be focused on the correlation quantification in interval process 
1 shown in Fig. 4, assuming that $m$ sample functions $x^{(p)}(t), p=1,2, \ldots, m$ of the interval

2 process $X^{I}(t)$ are available, based on which the self-correlation function can be defined as

3 follows.

4 Definition 4. For an interval process $X^{I}(t)$, the self-correlation function between any two 5 interval variables $X^{I}\left(t_{i}\right)$ and $X^{I}\left(t_{j}\right)$ at different times $t_{i}$ and $t_{j}$ is defined as $6 r_{X X}\left(t_{i}, t_{j}\right)$,

$$
r_{X X}\left(t_{i}, t_{j}\right)=\frac{\frac{1}{m} \sum_{p=1}^{m}\left(x_{i}^{(p)}-X_{i}^{c}\right)\left(x_{j}^{(p)}-X_{j}^{c}\right)}{W_{i}^{X} W_{j}^{X}}
$$

8 where $W_{i}^{X}=\sqrt{\frac{1}{m} \sum_{p=1}^{m}\left(x_{i}^{(p)}-X_{i}^{c}\right)^{2}}$ and $W_{j}^{X}=\sqrt{\frac{1}{m} \sum_{p=1}^{m}\left(x_{j}^{(p)}-X_{j}^{c}\right)^{2}} \cdot x_{i}^{(p)}$ is the value of the $9 p^{\text {th }}$ sample function at time $t_{i}$, and $X_{i}^{c}=X^{c}\left(t_{i}\right)$ is the value of middle point function of $X^{I}(t)$ at time $t_{i}$. The self-correlation function indicates the degree of correlation between an interval process $X^{I}(t)$ and itself at arbitrary different instants. Generally without causing confusion we can also denote $r_{X X}\left(t_{i}, t_{j}\right)$ as $r_{i j}$ in brief.

From Cauchy-Schwarz inequality [43] it is not difficult to derive that $-1 \leq r_{X X}\left(t_{i}, t_{j}\right) \leq 1$.

For the interval variable $X^{I}\left(t_{i}\right)$, there is $r_{X X}\left(t_{i}, t_{i}\right)=1$. Several groups of sample points of $\left(X^{I}\left(t_{i}\right), X^{I}\left(t_{j}\right)\right)$ and corresponding values of the self-correlation function are provided in Fig. 5, which show that the self-correlation function defined in Eq. (5) can well reflect the correlation between the uncertain variables of the process at any two time points. With the correlation quantified by the above method, the independent interval process can be defined as follows.

Definition 5. An interval process $X^{I}(t), t \in T$ is an independent process if for any two time points $t_{i}, t_{j} \in T, t_{i} \neq t_{j}$ there is $r_{X X}\left(t_{i}, t_{j}\right) \rightarrow 0$ when the number of the sample functions 
1 approaches to positive infinity, namely,

2

3 Otherwise, it is classified as a dependent interval process. To some degree, the independent

4 interval process is similar to the white noise process in traditional stochastic process theory.

5 When multiple uncertain processes are involved, the mutual-correlation function can be

7 Definition 6. For interval processes $X^{I}(t)$ and $Y^{I}(t), t \in T$, the mutual-correlation defined between different processes in a similar way.

function of $X^{I}\left(t_{i}\right)$ and $Y^{I}\left(t_{j}\right)$ is defined as $r_{X Y}\left(t_{i}, t_{j}\right)$,

9

$$
r_{X Y}\left(t_{i}, t_{j}\right)=\frac{\sum_{p=1}^{m}\left(x_{i}^{(p)}-X_{i}^{c}\right)\left(y_{j}^{(p)}-Y_{j}^{c}\right)}{\sqrt{\sum_{p=1}^{m}\left(x_{i}^{(p)}-X_{i}^{c}\right)^{2}} \sqrt{\sum_{p=1}^{m}\left(y_{j}^{(p)}-Y_{j}^{c}\right)^{2}}}
$$

where $x$ and $y$ stand for the sample functions of the interval processes $X^{I}(t)$ and $Y^{I}(t)$, respectively.

Notice that generally there is $r_{X Y}\left(t_{i}, t_{j}\right) \neq r_{Y X}\left(t_{i}, t_{j}\right)$. The reason is that $r_{X Y}\left(t_{i}, t_{j}\right)$ indicates the correlation between the interval processes $X^{I}(t)$ at time $t=t_{i}$ and $Y^{I}(t)$ at time $t=t_{j}$, while $r_{Y X}\left(t_{i}, t_{j}\right)$ indicates that between $X^{I}(t)$ at time $t=t_{j}$ and $Y^{I}(t)$ at time $t=t_{i}$.

\subsection{Stationary interval process}

Among various kinds of uncertain processes, those that their uncertainty characteristics do not change with time are generally called as stationary process. Ocean wave oscillations, amplitude of seismic wave in the period of strong earthquake and noises generated by machines etc., can be generally treated as stationary processes in practical engineering. 
1 Stationary processes play a primary role in practical structural dynamic analysis, since they

2 are widely encountered in engineering. In the following, the definition of stationary interval

3 process is also given.

4 Definition 7. An interval process $X^{I}(t), t \in T$ is stationary if the following two conditions

5 are satisfied,

6 (1) the middle point function $X^{c}(t)$ and the half width function $X^{r}(t)$ of the interval $7 \quad$ process are both constant;

8 (2) convergence in probability of the difference between $r_{X X}(t, t+\tau)$ and $r_{X X}(0, \tau)$, namely, 9 the probability, as $m$ goes to $+\infty$.

The first condition conveys that the upper and the lower bounds are both constants, as shown in Fig. 6. The second condition conveys that for arbitrary $t \in T$, the value of the self-correlation function between $X^{I}(t)$ and $X^{I}(t+\tau)$ at $t$ and $t+\tau$ approaches that between $X^{I}(0)$ and $X^{I}(\tau)$, with the number $m$ of the sample functions approaching positive infinity, namely,

$$
r_{X X}(t, t+\tau) \stackrel{m \rightarrow+\infty}{\longrightarrow} r_{X X}(0, \tau)=r(\tau)
$$

From this we can derive that the self-correlation function of a stationary interval process with a temporal distance $\tau$ are of the same value. In other words, the self-correlation function $r(\tau)$ is a function only with respect to the time interval $\tau$. Several typical forms of self-correlation function of a stationary interval process are listed in Table 1. In the left column, the forms of these self-correlation functions and the degree of 
correlation with regards to temporal distance $\tau$ are provided or plotted. In the right column, three sample curves are generated for each kind of self-correlation function. The upper and lower bounds of the interval processes are fixed. From the samples we can see, different self-correlation functions can have significant impacts on the shapes of the sample curves of an interval process. In other words, although the upper and lower bounds of two interval processes may be the same, they can differ a lot if their self-correlation functions are different.

Nevertheless, all the sample curves are within the predetermined upper and lower bounds, which agrees well with the definition of interval process model.

\subsection{Transformation of the interval process}

In the above sections, the interval process model has been proposed for time-varying uncertainty analysis. However, in most cases especially for those engineering problems with relatively high nonlinearity and large dimensions, corresponding uncertainty analyses such as computation of time-varying reliability will be complicated when the input parameters are dependent processes. To reduce the complexity and improve the computational efficiency in structural uncertainty analysis, a dependent interval process actually can be transformed into an independent one firstly. Hence in the following, a method will be given to realize such a transformation.

In many practical situations, a time-varying uncertain parameter can be approximately described by a sequence of variables that derived at certain time points [44, 45]. For $n$ selected time points $t_{1}, t_{2}, \ldots, t_{n}$, the interval process $X^{I}(t)$ correspondingly exhibits as $n$ interval variables $X^{I}\left(t_{1}\right), X^{I}\left(t_{2}\right), \ldots, X^{I}\left(t_{n}\right)$. When $n$ is sufficiently large and the time interval 

describe the varying characteristics of the interval process. Through the self-correlation

3 function $r_{X X}$, an $n \times n$ correlation matrix $\mathbf{R}_{X}$ can be created for the $n$-dimensional interval $4 \quad$ vector $\mathbf{X}^{I}=\left[\begin{array}{llll}X^{I}\left(t_{1}\right) & X^{I}\left(t_{2}\right) & \ldots & X^{I}\left(t_{n}\right)\end{array}\right]^{\mathrm{T}}$,

$$
\mathbf{R}_{X}=\left[\begin{array}{cccc}
r_{11} & r_{12} & \ldots & r_{1 n} \\
r_{21} & r_{22} & \ldots & r_{2 n} \\
\vdots & \vdots & \ddots & \vdots \\
r_{n 1} & r_{n 2} & \ldots & r_{n n}
\end{array}\right]=\left[\begin{array}{cccc}
r_{11} & r_{12} & \ldots & r_{1 n} \\
r_{12} & r_{22} & \ldots & r_{2 n} \\
\vdots & \vdots & \ddots & \vdots \\
r_{1 n} & r_{2 n} & \ldots & r_{n n}
\end{array}\right]
$$

where $r_{i j}=r_{X X}\left(t_{i}, t_{j}\right)$.

In practical problems, the correlation matrix is generally positive definite. To eliminate the correlation among the interval variables $X^{I}\left(t_{1}\right), X^{I}\left(t_{2}\right), \ldots, X^{I}\left(t_{n}\right)$ and hence obtain an independent process, the Cholesky decomposition [46] is employed in our formulation.

Lemma 1. Denote the interval vector of an interval process $X^{I}(t)$ as $\mathbf{X}^{I}=\left[\begin{array}{llll}X^{I}\left(t_{1}\right) & X^{I}\left(t_{2}\right) & \ldots & X^{I}\left(t_{n}\right)\end{array}\right]^{\mathrm{T}}$, and its correlation matrix as $\mathbf{R}_{X}$. Let $\mathbf{L}=\mathbf{W}_{X} \mathbf{L}_{X}$, where $\mathbf{L}_{X}$ is a lower triangular matrix derived from the Cholesky decomposition of the correlation matrix $\mathbf{R}_{X}$, and $\mathbf{W}_{X}$ is a diagonal matrix with diagonal entries $W_{i}^{X}, i=1,2, \ldots, n$ given in Definition 4. Denote the interval vector of another interval process $Y^{I}(t)$ as $\quad \mathbf{Y}^{I}=\left[\begin{array}{lllll}Y^{I}\left(t_{1}\right) & Y^{I}\left(t_{2}\right) & \ldots & Y^{I}\left(t_{n}\right)\end{array}\right]^{\mathrm{T}}$, and let $\mathbf{Y}^{I}=\mathbf{L}^{-1} \mathbf{X}^{I}$, then the correlation matrix $\mathbf{R}_{Y}$ of the interval vector $\mathbf{Y}^{I}$ will be an identity matrix,

$$
\mathbf{R}_{Y}=\mathbf{I}
$$

The proof is given in Appendix A.

Lemma 1 indicates that the correlation between each pair of interval variables $Y_{i}$ and $Y_{j}$ $(i \neq j)$ is zero, thus $Y^{I}(t)$ can be approximately regarded as an independent interval process. Based on this, the variation ranges of $Y_{i}, i=1,2, \ldots, n$ can be also obtained as follows. 
1 Lemma 2. For the interval sequences $X_{i}^{I}=\left[X_{i}^{L}, X_{i}^{U}\right]$ and $Y_{i}^{I}=\left[Y_{i}^{L}, Y_{i}^{U}\right], \quad i=1,2, \ldots, n$,

2 through the above transformation the mapping relation between $\mathbf{X}^{I}$ and $\mathbf{Y}^{I}$ can be created 3 as,

4

5

$$
\begin{gathered}
\mathbf{Y}^{c}=\mathbf{L}^{-1} \mathbf{X}^{c} \\
\mathbf{Y}^{r}=[|\mathbf{L}|]^{-1} \mathbf{X}^{r}
\end{gathered}
$$

where $\mathbf{X}^{c}$ and $\mathbf{Y}^{c}$ are middle point vectors, $\mathbf{X}^{r}$ and $\mathbf{Y}^{r}$ are half width vectors, and $[|\mathbf{L}|]$ denotes the matrix whose entries are absolute values of corresponding ones in the matrix $\mathbf{L}$. The proof for Lemma 2 is given in Appendix B.

Thus, we can obtain a new interval process $Y^{I}(t)$, which can be used as an independent process. With such a transformation, the subsequent uncertainty analyses can be carried out more conveniently and efficiently.

\section{Non-random vibration analysis method}

Dynamic analysis of structures under time-varying inputs such as vibration of tall buildings under wind forces, vibration of high speed automobiles subjected to random road excitations, are widely encountered by engineers. In traditional random vibration theory, the time-varying input parameters of the system such as load excitations are modelled as stochastic processes, and hence the structural responses can also be described as stochastic processes with characteristics in probabilistic terms. So far a multitude of researches and achievements have been gained in this area [47-52]. While as stated previously, random vibration analysis generally requires relatively large number of experimental samples, and it may face difficulty for many practical engineering problems that only limited samples are available. 

method for structures subjected to time-varying or dynamic uncertain loads, namely, the

3 "non-random vibration analysis". In this method, the input time-varying uncertain parameters

4 are modelled as interval processes, and the output structural responses are formulated in the

5 form of upper and lower bounds, which can be referred to as an important guidance for

6 practical safety analysis and reliability design of structures or products. The non-random

7 vibration method is generally applicable to dynamic analyses of many complicated structures

8 where experimental samples are difficult or costly to obtain, thus providing a beneficial 9 complement for the existing random vibration theory.

\subsection{Single degree of freedom (SDOF) vibration system}

In this part, we firstly introduce the traditional vibration analysis of SDOF system under

12

\subsubsection{Under deterministic excitation}

stiffness $k$, damping coefficient $c$, and external force $F(t)$. By using Newton's law, the equation of motion in terms of the displacement $z$, velocity $\dot{z}$, and acceleration $\ddot{z}$ can be given in the form [53],

$$
m \ddot{z}+c \dot{z}+k z=F(t)
$$

or 


$$
\ddot{z}+2 \zeta \omega_{n} \dot{z}+\omega_{n}^{2} z=\frac{F(t)}{m}
$$

where the natural frequency $\omega_{n}$ and damping ratio $\zeta$ are given by,

$$
\begin{aligned}
\omega_{n} & =\sqrt{k / m} \\
c_{\mathrm{c}} & =2 m \omega_{n} \\
\zeta & =\frac{c}{c_{\mathrm{c}}}
\end{aligned}
$$

4 In the above equation, $c_{\mathrm{c}}$ is the critical damping coefficient.

5 In many practical problems the analytic expression of the excitation $F(t)$ is not available.

6 Very often only a sequence of values $F\left(t_{i}\right)$ (or denoted as $F_{i}$ ) can be obtained at instants

$7 t_{i}, i=1,2, \ldots, n$. Herein the Piecewise Linear Interpolation function [54] is firstly used to

8 approximately fit the sequence of $F_{i}$, where the rate of change of $F(t)$ within arbitrary

9 period of time is supposed to be linear, as shown in Fig. 8. In this way, Duhamel's integral [55]

10 can be then applied to compute the response at time $t=t_{j}$ [56],

11

$$
\begin{aligned}
z_{j}= & \frac{\Delta F_{j}}{k \Delta t_{j}}\left[\Delta t_{j}-\frac{2 \zeta}{\omega_{n}}+\mathrm{e}^{-\zeta \omega_{n} \Delta t_{j}}\left\{\frac{2 \zeta}{\omega_{n}} \cos \omega_{\mathrm{d}} \Delta t_{j}-\frac{\omega_{\mathrm{d}}^{2}-\zeta^{2} \omega_{n}^{2}}{\omega_{n}^{2} \omega_{\mathrm{d}}} \sin \omega_{\mathrm{d}} \Delta t_{j}\right\}\right] \\
& +\frac{F_{j-1}}{k}\left[1-\mathrm{e}^{-\zeta \omega_{n} \Delta t_{j}}\left\{\cos \omega_{\mathrm{d}} \Delta t_{j}+\frac{\zeta \omega_{n}}{\omega_{\mathrm{d}}} \sin \omega_{\mathrm{d}} \Delta t_{j}\right\}\right] \\
& +\mathrm{e}^{-\zeta \omega_{n} \cdot \Delta t_{j}}\left[x_{j-1} \cos \omega_{\mathrm{d}} \Delta t_{j}+\frac{\dot{x}_{j-1}+\zeta \omega_{n} x_{j-1}}{\omega_{\mathrm{d}}} \sin \omega_{\mathrm{d}} \Delta t_{j}\right]
\end{aligned}
$$

12 where $\Delta F_{j}=F_{j}-F_{j-1}, \Delta t_{j}=t_{j}-t_{j-1}, \omega_{\mathrm{d}}=\sqrt{1-\zeta^{2}} \omega_{n}$. The velocity $\dot{z}_{j}$ at time $t=t_{j}$ can be calculated as follows,

$$
\begin{aligned}
\dot{z}_{j}= & \frac{\Delta F_{j}}{k \Delta t_{j}}\left[1-\mathrm{e}^{-\zeta \omega_{n} \Delta t_{j}}\left\{\cos \omega_{\mathrm{d}} \Delta t_{j}+\frac{\zeta \omega_{n}}{\omega_{\mathrm{d}}} \sin \omega_{\mathrm{d}} \Delta t_{j}\right\}\right] \\
& +\frac{F_{j-1}}{k} \mathrm{e}^{-\zeta \omega_{n} \Delta t_{j}} \frac{\omega_{n}^{2}}{\omega_{\mathrm{d}}} \sin \omega_{\mathrm{d}} \Delta t_{j} \\
& +\mathrm{e}^{-\zeta \omega_{n} \cdot \Delta t_{j}}\left[\dot{z}_{j-1} \cos \omega_{\mathrm{d}} \Delta t_{j}-\frac{\zeta \omega_{n}}{\omega_{\mathrm{d}}}\left(\dot{z}_{j-1}+\frac{\omega_{n}}{\zeta} z_{j-1}\right) \sin \omega_{\mathrm{d}} \Delta t_{j}\right]
\end{aligned}
$$

Equations (17) and (18) can be rewritten in the following form, 


$$
\begin{aligned}
& z_{j}=A_{j} \Delta F_{j}+B_{j} F_{j-1}+C_{j} z_{j-1}+D_{j} \dot{z}_{j-1} \\
& \dot{z}_{j}=\bar{A}_{j} \Delta F_{j}+\bar{B}_{j} F_{j-1}+\bar{C}_{j} z_{j-1}+\bar{D}_{j} \dot{z}_{j-1}
\end{aligned}
$$

2 where,

$$
\begin{aligned}
& A_{j}=\frac{1}{k \Delta t_{j}}\left[\Delta t_{j}-\frac{2 \zeta}{\omega_{n}}+\mathrm{e}^{-\zeta \omega_{n} \Delta t_{j}}\left\{\frac{2 \zeta}{\omega_{n}} \cos \omega_{\mathrm{d}} \Delta t_{j}-\frac{\omega_{\mathrm{d}}^{2}-\zeta^{2} \omega_{n}^{2}}{\omega_{n}^{2} \omega_{\mathrm{d}}} \sin \omega_{\mathrm{d}} \Delta t_{j}\right\}\right] \\
& B_{j}=\frac{1}{k}\left[1-\mathrm{e}^{-\zeta \omega_{n} \Delta t_{j}}\left\{\cos \omega_{\mathrm{d}} \Delta t_{j}+\frac{\zeta \omega_{n}}{\omega_{\mathrm{d}}} \sin \omega_{\mathrm{d}} \Delta t_{j}\right\}\right] \\
& C_{j}=\mathrm{e}^{-\zeta \omega_{n} \Delta t_{j}}\left(\cos \omega_{\mathrm{d}} \Delta t_{j}+\frac{\zeta \omega_{n}}{\omega_{\mathrm{d}}} \sin \omega_{\mathrm{d}} \Delta t_{j}\right) \\
& D_{j}=\mathrm{e}^{-\zeta \omega_{n} \Delta t_{j}} \frac{1}{\omega_{\mathrm{d}}} \sin \omega_{\mathrm{d}} \Delta t_{j} \\
& \bar{A}_{j}=\frac{1}{k \Delta t_{j}}\left[1-\mathrm{e}^{-\zeta \omega_{n} \Delta t_{j}}\left\{\cos \omega_{\mathrm{d}} \Delta t_{j}+\frac{\zeta \omega_{n}}{\omega_{\mathrm{d}}} \sin \omega_{\mathrm{d}} \Delta t_{j}\right\}\right] \\
& \bar{B}_{j}=\frac{1}{k} \mathrm{e}^{-\zeta \omega_{n} \Delta t_{j}} \frac{\omega_{n}^{2}}{\omega_{\mathrm{d}}} \sin \omega_{\mathrm{d}} \Delta t_{j} \\
& \bar{C}_{j}=-\mathrm{e}^{-\zeta \omega_{n} \Delta t_{j}} \frac{\omega_{n}^{2}}{\omega_{\mathrm{d}}} \sin \omega_{\mathrm{d}} \Delta t_{j} \\
& \bar{D}_{j}=\mathrm{e}^{-\zeta \omega_{n} \Delta t_{j}}\left(\cos \omega_{\mathrm{d}} \Delta t_{j}-\frac{\zeta \omega_{n}}{\omega_{\mathrm{d}}} \sin \omega_{\mathrm{d}} \Delta t_{j}\right)
\end{aligned}
$$

4 Equation (19) can be also expressed in the following matrix form,

5

$$
\left[\begin{array}{c}
z_{j} \\
\dot{z}_{j}
\end{array}\right]=\mathbf{H}\left[\begin{array}{c}
\mathbf{F} \\
\mathbf{z}_{0}
\end{array}\right]
$$

6 where $\mathbf{H}$ is a $2 \times(j+3)$ constant coefficient matrix that can be constructed by the recursive

7 procedure in Appendix C. $\mathbf{F}=\left[\begin{array}{llllll}F_{j} & F_{j-1} & \ldots & F_{1} & F_{0}\end{array}\right]^{\mathrm{T}}$ is the vector of force sequence, and $8 \quad \mathbf{z}_{0}=\left[\begin{array}{ll}z_{0} & \dot{z}_{0}\end{array}\right]^{\mathrm{T}}$ is the initial condition.

9 In the above analysis, Duhamel's integral [55] is adopted to calculate the structural responses such as displacement and velocity. We can also adopt other related numerical methods such as Newmark Method, Wilson- $\theta$ Method, Central Difference Method (CDM), Houbolt Method, etc. [57], and a similar result such as Eq. (21) can be also obtained. 
2 In our non-random vibration analysis, the excitation force is treated as an interval process

$3 F^{I}(t)=\left[F^{L}(t), F^{U}(t)\right]$ with the self-correlation function $r(t, \tau)$ (or $r(\tau)$ for a stationary

4 process). Namely for arbitrary time point $t_{i}$, the possible values of the uncertain quantity

$5 \quad F\left(t_{i}\right)$ belong to an interval $\left[F^{L}\left(t_{i}\right), F^{U}\left(t_{i}\right)\right]$, i.e., $F\left(t_{i}\right) \in\left[F^{L}\left(t_{i}\right), F^{U}\left(t_{i}\right)\right]$. Through

6 discretizing the process, $F^{I}(t)$ can be represented by an interval vector

$7 \quad \mathbf{F}^{I}=\left[\begin{array}{lllll}F_{j}^{I} & F_{j-1}^{I} & \ldots & F_{1}^{I} & F_{0}^{I}\end{array}\right]^{\mathrm{T}}$. Hence after introducing the interval process Eq. (21) can be

8 rewritten as,

9

$$
\left[\begin{array}{c}
z_{j} \\
\dot{z}_{j}
\end{array}\right]=\mathbf{H}\left[\begin{array}{c}
\mathbf{F}^{I} \\
\mathbf{z}_{0}
\end{array}\right]
$$

By using the transformation in section 2.4 , the dependent interval process $F^{I}(t)$ can be firstly changed to an independent process $\bar{F}^{I}(t)$. A corresponding interval vector $\overline{\mathbf{F}}^{I}$ can be obtained by $\overline{\mathbf{F}}^{I}=\mathbf{L}^{-1} \mathbf{F}^{I}$, herein $\mathbf{L}$ is a $(j+1) \times(j+1)$ lower triangular matrix derived from the correlation matrix (see Lemma 1). Through this treatment, Eq. (22) can be rewritten as,

$$
\left[\begin{array}{c}
z_{j} \\
\dot{z}_{j}
\end{array}\right]=\mathbf{H}\left[\begin{array}{c}
\mathbf{L} \overline{\mathbf{F}}^{I} \\
\mathbf{z}_{0}
\end{array}\right]=\left[\begin{array}{ll}
\mathbf{H}_{\mathbf{F}} & \mathbf{H}_{\mathbf{z}}
\end{array}\right]\left[\begin{array}{c}
\mathbf{L} \overline{\mathbf{F}}^{I} \\
\mathbf{z}_{0}
\end{array}\right]=\mathbf{H}_{\mathbf{F}} \mathbf{L} \overline{\mathbf{F}}^{I}+\mathbf{H}_{\mathbf{z}} \mathbf{z}_{0}
$$

where $\mathbf{H}_{\mathbf{F}}$ and $\mathbf{H}_{\mathbf{z}}$ are block matrices of $\mathbf{H}$, with dimensions $2 \times(j+1)$ and $2 \times 2$, respectively. Denote $\overline{\mathbf{F}}^{I}=\left[\overline{\mathbf{F}}^{c}-\overline{\mathbf{F}}^{r}, \overline{\mathbf{F}}^{c}+\overline{\mathbf{F}}^{r}\right]$, then from Eqs. (12) and (13) there is,

$$
\overline{\mathbf{F}}^{c}=\mathbf{L}^{-1} \mathbf{F}^{c}
$$

$$
\overline{\mathbf{F}}^{r}=[|\mathbf{L}|]^{-1} \mathbf{F}^{r}
$$

where $\overline{\mathbf{F}}^{c}$ and $\overline{\mathbf{F}}^{r}$ are middle point vector and half width vector of the transformed interval vector $\overline{\mathbf{F}}^{I}$, respectively. Denote $[\boldsymbol{\delta}] \overline{\mathbf{F}}^{r}$ as,

$$
[\boldsymbol{\delta}] \overline{\mathbf{F}}^{r}=\left[\begin{array}{llll}
\delta_{j}^{I} \bar{F}_{j}^{r} & \ldots & \delta_{1}^{I} \bar{F}_{1}^{r} & \delta_{0}^{I} \bar{F}_{0}^{r}
\end{array}\right]^{\mathrm{T}}, \delta_{i}^{I}=[-1,1], i=0,1, \ldots, j
$$


1

3 Substituting Eqs. (27), (24) and (25) into Eq. (23) yields,
4

$$
\begin{aligned}
{\left[\begin{array}{c}
z_{j} \\
\dot{z}_{j}
\end{array}\right] } & =\mathbf{H}_{\mathbf{F}} \mathbf{L} \overline{\mathbf{F}}^{I}+\mathbf{H}_{\mathbf{z}} \mathbf{z}_{0} \\
& =\mathbf{H}_{\mathbf{F}} \mathbf{L} \overline{\mathbf{F}}^{c}+\mathbf{H}_{\mathbf{F}} \mathbf{L}[\boldsymbol{\delta}] \overline{\mathbf{F}}^{r}+\mathbf{H}_{\mathbf{z}} \mathbf{z}_{0} \\
& \in\left[\left(\mathbf{H}_{\mathbf{F}} \mathbf{L} \overline{\mathbf{F}}^{c}+\mathbf{H}_{\mathbf{z}} \mathbf{z}_{0}\right)-\left[\left|\mathbf{H}_{\mathbf{F}} \mathbf{L}\right|\right] \overline{\mathbf{F}}^{r}, \quad\left(\mathbf{H}_{\mathbf{F}} \mathbf{L} \overline{\mathbf{F}}^{c}+\mathbf{H}_{\mathbf{z}} \mathbf{z}_{0}\right)+\left[\left|\mathbf{H}_{\mathbf{F}} \mathbf{L}\right|\right] \overline{\mathbf{F}}^{r}\right] \\
& =\left[\left(\mathbf{H}_{\mathbf{F}} \mathbf{L} \mathbf{L}^{-1} \mathbf{F}^{c}+\mathbf{H}_{\mathbf{z}} \mathbf{z}_{0}\right)-\left[\left|\mathbf{H}_{\mathbf{F}} \mathbf{L}\right|\right][|\mathbf{L}|]^{-1} \mathbf{F}^{r}, \quad\left(\mathbf{H}_{\mathbf{F}} \mathbf{L} \mathbf{L}^{-1} \mathbf{F}^{c}+\mathbf{H}_{\mathbf{z}} \mathbf{z}_{0}\right)+\left[\left|\mathbf{H}_{\mathbf{F}} \mathbf{L}\right|\right][|\mathbf{L}|]^{-1} \mathbf{F}^{r}\right] \\
& =\left[\left(\mathbf{H}_{\mathbf{F}} \mathbf{F}^{c}+\mathbf{H}_{\mathbf{z}} \mathbf{z}_{0}\right)-\left[\left|\mathbf{H}_{\mathbf{F}} \mathbf{L}\right|\right][|\mathbf{L}|]^{-1} \mathbf{F}^{r}, \quad\left(\mathbf{H}_{\mathbf{F}} \mathbf{F}^{c}+\mathbf{H}_{\mathbf{z}} \mathbf{z}_{0}\right)+\left[\left|\mathbf{H}_{\mathbf{F}} \mathbf{L}\right|\right][|\mathbf{L}|]^{-1} \mathbf{F}^{r}\right]
\end{aligned}
$$

This implies that the upper and lower bounds of the responses at time $t=t_{j}$ are,

$$
\begin{aligned}
& \mathbf{z}_{j}^{U}=\left[\begin{array}{c}
z_{j}^{U} \\
\dot{z}_{j}^{U}
\end{array}\right]=\left(\mathbf{H}_{\mathbf{F}} \mathbf{F}^{c}+\mathbf{H}_{\mathbf{z}} \mathbf{z}_{0}\right)+\left[\left|\mathbf{H}_{\mathbf{F}} \mathbf{L}\right|\right][|\mathbf{L}|]^{-1} \mathbf{F}^{r} \\
& \mathbf{z}_{j}^{L}=\left[\begin{array}{c}
z_{j}^{L} \\
\dot{z}_{j}^{L}
\end{array}\right]=\left(\mathbf{H}_{\mathbf{F}} \mathbf{F}^{c}+\mathbf{H}_{\mathbf{z}} \mathbf{z}_{0}\right)-\left[\left|\mathbf{H}_{\mathbf{F}} \mathbf{L}\right|\right][|\mathbf{L}|]^{-1} \mathbf{F}^{r}
\end{aligned}
$$

\subsection{Multiple degree of freedom (MDOF) vibration system}

In practical engineering, most vibration systems are of multiple degrees of freedom (MDOF). Hence in the following the non-random vibration analysis for MDOF systems will be also formulated. Similarly, we will first discuss the case that the forces are deterministic, and then the one that the forces are uncertain. 


\subsubsection{Under deterministic excitations}

2

9 Firstly the external excitations $\mathbf{F}$ are assumed as deterministic. The Central Difference

$$
\left[\frac{1}{(\Delta t)^{2}} \mathbf{M}+\frac{1}{2 \Delta t} \mathbf{C}\right] \mathbf{Z}\left(t_{j+1}\right)=\mathbf{F}\left(t_{j}\right)-\left[\mathbf{K}-\frac{2}{(\Delta t)^{2}} \mathbf{M}\right] \mathbf{Z}\left(t_{j}\right)-\left[\frac{1}{(\Delta t)^{2}} \mathbf{M}-\frac{1}{2 \Delta t} \mathbf{C}\right] \mathbf{Z}\left(t_{j-1}\right)
$$

$$
\mathbf{Z}=\left[\begin{array}{c}
z_{1}(t) \\
z_{2}(t) \\
\vdots \\
z_{N}(t)
\end{array}\right], \quad \dot{\mathbf{Z}}=\left[\begin{array}{c}
\dot{z}_{1}(t) \\
\dot{z}_{2}(t) \\
\vdots \\
\dot{z}_{N}(t)
\end{array}\right], \quad \quad \ddot{\mathbf{Z}}=\left[\begin{array}{c}
\ddot{z}_{1}(t) \\
\ddot{z}_{2}(t) \\
\vdots \\
\ddot{z}_{N}(t)
\end{array}\right], \quad \mathbf{F}=\left[\begin{array}{c}
F_{1}(t) \\
F_{2}(t) \\
\vdots \\
F_{N}(t)
\end{array}\right]
$$

where $\mathbf{M}, \mathbf{C}$ and $\mathbf{K}$ are $N \times N$ Mass Matrix, Damping Matrix and Stiffness Matrix, respectively; $\mathbf{Z}, \dot{\mathbf{Z}}, \ddot{\mathbf{Z}}$ and $\mathbf{F}$ denote $N$-dimensional displacement, velocity, acceleration and external excitation vectors, respectively,

Method (CDM) [57] can be employed to solve Eq. (31). By CDM, the velocity and acceleration vectors at time $t_{j}$ are given as,

$$
\begin{gathered}
\dot{\mathbf{Z}}\left(t_{j}\right)=\frac{\mathbf{Z}\left(t_{j+1}\right)-\mathbf{Z}\left(t_{j-1}\right)}{2 \Delta t} \\
\ddot{\mathbf{Z}}\left(t_{j}\right)=\frac{\mathbf{Z}\left(t_{j+1}\right)-2 \mathbf{Z}\left(t_{j}\right)+\mathbf{Z}\left(t_{j-1}\right)}{(\Delta t)^{2}}
\end{gathered}
$$

$\Delta t=t_{j}-t_{j-1}$ is the temporal distance between two adjacent time points. Substituting Eq. (33) into Eq. (31) at time $t_{j}$, the following equation for computation of the displacement vector $\mathbf{Z}\left(t_{j+1}\right)$ can be derived, The CDM is conditionally stable and the condition is $\Delta t \leq \Delta t_{\mathrm{cr}}=T_{n} / \pi$, where $T_{n}$ denotes the minimum natural period of the system. The procedure of CDM can be concluded as 
2 1) Calculate $\ddot{\mathbf{Z}}\left(t_{0}\right)$ with the following equation,

3

4

5

$$
\mathbf{M} \ddot{\mathbf{Z}}\left(t_{0}\right)=\mathbf{F}\left(t_{0}\right)-\mathbf{C} \dot{\mathbf{Z}}\left(t_{0}\right)-\mathbf{K Z}\left(t_{0}\right)
$$

2) Select an appropriate time step $\Delta t \leq \Delta t_{\mathrm{cr}}$, and obtain the following constants,

$$
a_{0}=\frac{1}{(\Delta t)^{2}}, \quad a_{1}=\frac{1}{2 \Delta t}, \quad a_{2}=2 a_{0}, \quad a_{3}=\frac{1}{a_{2}}
$$

3) Calculate $\mathbf{Z}\left(t_{-1}\right)$ by the following equation,

$$
\mathbf{Z}\left(t_{-1}\right)=\mathbf{Z}\left(t_{0}\right)-\dot{\mathbf{Z}}\left(t_{0}\right) \Delta t+a_{3} \ddot{\mathbf{Z}}\left(t_{0}\right)
$$

4) Calculate the equivalent Mass Matrix $\hat{\mathbf{M}}$,

$$
\hat{\mathbf{M}}=a_{0} \mathbf{M}+a_{1} \mathbf{C}
$$

5) At each time point, calculate

I) The equivalent force $\hat{\mathbf{F}}\left(t_{j}\right)$,

$$
\hat{\mathbf{F}}\left(t_{j}\right)=\mathbf{F}\left(t_{j}\right)-\left(\mathbf{K}-a_{2} \mathbf{M}\right) \mathbf{Z}\left(t_{j}\right)-\left(a_{0} \mathbf{M}-a_{1} \mathbf{C}\right) \mathbf{Z}\left(t_{j-1}\right)
$$

II) Displacement vector $\mathbf{Z}\left(t_{j+1}\right)$ at time $t_{j+1}$, which can be derived from Eq. (35) and Eq. (40),

15

$$
\dot{\mathbf{Z}}\left(t_{j}\right)=a_{1}\left(\mathbf{Z}\left(t_{j+1}\right)-2 \mathbf{Z}\left(t_{j}\right)+\mathbf{Z}\left(t_{j-1}\right)\right)
$$$$
\ddot{\mathbf{Z}}\left(t_{j}\right)=a_{0}\left(\mathbf{Z}\left(t_{j+1}\right)-\mathbf{Z}\left(t_{j-1}\right)\right)
$$

III) Velocity vector $\dot{\mathbf{Z}}\left(t_{j}\right)$ and acceleration vector $\ddot{\mathbf{Z}}\left(t_{j}\right)$ at time $t_{j}$, 


$$
\mathbf{Z}\left(t_{j+1}\right)=\sum_{k=1}^{N} \hat{\mathbf{H}}_{\mathbf{F}_{k}}^{*} \mathbf{F}_{k}+\hat{\mathbf{H}}_{\mathbf{Z}}\left[\begin{array}{l}
\mathbf{Z}\left(t_{0}\right) \\
\dot{\mathbf{Z}}\left(t_{0}\right)
\end{array}\right]
$$

2 where the $N \times(j+1)$ matrix $\hat{\mathbf{H}}_{\mathbf{F}_{k}}^{*}$ and $N \times 2 N$ matrix $\hat{\mathbf{H}}_{\mathbf{Z}}$ can be derived from the

3 recursive procedure in Appendix D. $\quad \mathbf{F}_{k}=\left[F_{k}\left(t_{j}\right), F_{k}\left(t_{j-1}\right), \ldots, F_{k}\left(t_{0}\right)\right]^{\mathrm{T}} \quad$ is $\quad$ a

$4 \quad(j+1)$-dimensional force vector constituted by $F_{k}(t)$ at certain time points, where $F_{k}(t)$ is

5 the $k$ th excitation force among the forces vector $\mathbf{F}$ denoted in Eq. (32). The $2 N$-dimensional

6 vector $\left[\begin{array}{l}\mathbf{Z}\left(t_{0}\right) \\ \dot{\mathbf{Z}}\left(t_{0}\right)\end{array}\right]$ means the initial conditions. Similarly, other numerical methods such as

7 Newmark Method, Wilson- $\theta$ Method etc. [57], can also be adopted to calculate the

8 displacements and velocities, and similar forms like Eq. (44) can be obtained.

\subsubsection{Under uncertain excitations}

Consider that the time-varying forces are uncertain and modelled as interval processes, i.e., $F_{k}^{I}(t)=\left[F_{k}^{L}(t), F_{k}^{U}(t)\right], \quad k=1,2, \ldots, N$. For convenience of analysis, here the forces are assumed to be independent with each other. Denote $\mathbf{F}_{k}^{I}$ as the vector form of the force $F_{k}^{I}(t)$, and $\mathbf{R}_{\mathbf{F}_{k}}$ as the correlation matrix of $\mathbf{F}_{k}^{I}$, then the displacements vector can be rewritten as,

$$
\mathbf{Z}\left(t_{j+1}\right)=\sum_{k=1}^{N} \hat{\mathbf{H}}_{\mathbf{F}_{k}}^{*} \mathbf{F}_{k}^{I}+\hat{\mathbf{H}}_{\mathbf{Z}}\left[\begin{array}{c}
\mathbf{Z}\left(t_{0}\right) \\
\dot{\mathbf{Z}}\left(t_{0}\right)
\end{array}\right]
$$

Similar to the SDOF system, firstly the transformation introduced in section 2.4 needs to be performed for each interval vector $\mathbf{F}_{k}^{I}$,

$$
\mathbf{L}_{k}=\mathbf{W}_{\mathbf{F}_{k}} \mathbf{L}_{\mathbf{F}_{k}}, \quad k=1,2, \ldots, N
$$

where $\mathbf{W}_{\mathbf{F}_{k}}$ is a $(j+1) \times(j+1)$ diagonal matrix with diagonal entries $W_{i}^{F_{k}}$, and $\mathbf{L}_{\mathbf{F}_{k}}$ is the decomposed lower triangular matrix of $\mathbf{R}_{\mathbf{F}_{k}}$, with dimension $(j+1) \times(j+1)$. Letting 
$1 \quad \overline{\mathbf{F}}_{k}^{I}=\mathbf{L}_{k}^{-1} \mathbf{F}_{k}^{I}$ and substituting it into Eq. (45) yields,

$$
\mathbf{Z}\left(t_{j+1}\right)=\sum_{k=1}^{N} \hat{\mathbf{H}}_{\mathbf{F}_{k}}^{*} \mathbf{L}_{k} \overline{\mathbf{F}}_{k}^{I}+\hat{\mathbf{H}}_{\mathbf{Z}}\left[\begin{array}{c}
\mathbf{Z}\left(t_{0}\right) \\
\dot{\mathbf{Z}}\left(t_{0}\right)
\end{array}\right]
$$

3 From Lemma 2, the middle points $\overline{\mathbf{F}}_{k}^{c}$ and half widths $\overline{\mathbf{F}}_{k}^{r}$ of $\overline{\mathbf{F}}_{k}^{I}$ can be derived as,

$$
\begin{gathered}
\overline{\mathbf{F}}_{k}^{c}=\mathbf{L}_{k}^{-1} \mathbf{F}_{k}^{c} \\
\overline{\mathbf{F}}_{k}^{r}=\left[\left|\mathbf{L}_{k}\right|\right]^{-1} \mathbf{F}_{k}^{r}
\end{gathered}
$$

Similar to the SDOF case, the displacement bounds for the MDOF system at time $t_{j+1}$ can be obtained,

$$
\begin{aligned}
& \mathbf{Z}^{U}\left(t_{j+1}\right)=\left(\sum_{k=1}^{N} \hat{\mathbf{H}}_{\mathbf{F}_{k}}^{*} \mathbf{F}_{k}^{c}+\hat{\mathbf{H}}_{\mathbf{z}}\left[\begin{array}{c}
\mathbf{Z}\left(t_{0}\right) \\
\dot{\mathbf{Z}}\left(t_{0}\right)
\end{array}\right]\right)+\sum_{k=1}^{N}\left[\left|\hat{\mathbf{H}}_{\mathbf{F}_{k}}^{*} \mathbf{L}_{k}\right|\right]\left[\left|\mathbf{L}_{k}\right|\right]^{-1} \mathbf{F}_{k}^{r} \\
& \mathbf{Z}^{L}\left(t_{j+1}\right)=\left(\sum_{k=1}^{N} \hat{\mathbf{H}}_{\mathbf{F}_{k}}^{*} \mathbf{F}_{k}^{c}+\hat{\mathbf{H}}_{\mathbf{z}}\left[\begin{array}{l}
\mathbf{Z}\left(t_{0}\right) \\
\dot{\mathbf{Z}}\left(t_{0}\right)
\end{array}\right]\right)-\sum_{k=1}^{N}\left[\left|\hat{\mathbf{H}}_{\mathbf{F}_{k}}^{*} \mathbf{L}_{k}\right|\right]\left[\left|\mathbf{L}_{k}\right|\right]^{-1} \mathbf{F}_{k}^{r}
\end{aligned}
$$

With the displacement bounds at each time point computed, the upper and lower dynamical displacement response bounds can be subsequently obtained. Besides, the velocity and acceleration bounds can also be derived by a similar procedure in Appendix D.

\section{Numerical examples and discussions}

In this section, several numerical examples are provided to illustrate the effectiveness of the proposed interval process model and the non-random vibration analysis method. Both SDOF and MDOF systems are investigated. 


\subsection{An SDOF system}

\subsection{1 $F^{I}(t)$ is stationary}

Consider the spring-mass-damper system with mass $m=1$, damping coefficient $c=0.2$ and spring stiffness $k=1$ subjected to a dynamic force $F(t)$, as shown in Fig. 7. Herein $F(t)$ is treated as a stationary interval process $F^{I}(t)$ with middle point function $F^{c}(t)=12$ and half width function $F^{r}(t)=3$. In other words, there is $F(t) \in[9,15]$ for arbitrary time $t$. The initial condition is $z(0)=\dot{z}(0)=0$. Herein the time step $\Delta t$ is set as $0.5 \mathrm{~s}$ for discretization of the process.

Firstly, effects of different types of self-correlation functions on the system response bounds are studied. The displacement and velocity responses are analyzed when the self-correlation function of $F(t)$ is assumed to be the following typical cases: (a) $r(\tau)=\mathrm{e}^{-\alpha|\tau|} ;$ (b) $r(\tau)=\mathrm{e}^{-\alpha|\tau|} \cos \omega \tau ;$ (c) $r(\tau)=1-|\tau| / T_{0} ;$ (d) $r(\tau)=0, \tau \neq 0$. Herein the parameters are set as $\alpha=0.2, \omega=\pi / 4, T_{0}=60 \mathrm{~s}$, and $\tau$ indicates the temporal distance. The response bounds of displacement $z(t)$ and velocity $\dot{z}(t)$ are plotted in Fig. 10 and Fig. 11, respectively. From Fig. 10, it can be observed that when $F^{I}(t)$ is an independent process (case (d), $r(\tau)=0, \tau \neq 0$ ), the displacement bounds of $z(t)$ exhibits a wider domain than those when $F^{I}(t)$ is a dependent process (cases (a)-(c)). The reason is that when the force $F^{I}(t)$ is an independent process, the possible values of the force at a certain instant will have no influence on those at other instants. In other words, no extra constraints are imposed on the forms and shapes of the time-varying process within its lower and upper bound functions. Therefore, more types of structural responses are possible to emerge in this case, thus causing 
1 a wider response bounds. From Fig. 10 (a)-(d), we also find that in all of the four correlation

2 cases, the response bounds of displacement $z(t)$ can be roughly divided into two stages,

3 namely, the transient response stage and the steady-state response stage. In the transient

4 response stage, the response bounds exhibit obvious oscillation, and the middle point and half

5 width functions both fluctuate with time. This kind of oscillation tends to be slighter with time,

6 and finally a steady state can be reached. In this stage, both the middle point and half width of

7 the responses keep nearly constant. This phenomenon is similar to the vibration of mechanical

8 systems under deterministic excitations, in which a steady-state response stage can be finally

9 reached for a damped system. To sum up the above analysis, the computational results could

tell us: 1) the self-correlation function of the time-varying force $F^{I}(t)$ may have a

11 significant impact on the dynamic responses such as displacement and velocity; 2) the

computed response bounds under the three cases of dependent interval process are all

enclosed by those under the independent process; 3) a steady-state response stage can be

reached for the damped vibration system after an initial transient response stage, which does not depend on whether the force $F^{I}(t)$ is a dependent or an independent process.

carried out when different self-correlation functions are designated for $F^{I}(t)$. The results are given in Fig. 12, which are quite different from those when the system is damped. It can be of self-correlation function, the response bounds become wider and wider over time and exhibit oscillation characteristics. Or in other words, the middle point functions of the response bounds are oscillatory, and the half widths get larger over time. However, the results 
1 of the undamped system shows that the response bounds under the dependent $F^{I}(t)$ are all

2 enclosed by those under the independent excitation, which is same as the damped case.

\section{$3 \quad 4.1 .2 \quad F^{I}(t)$ is non-stationary}

5

This case also focuses on the damped SDOF system in Fig. 7, and the values of relevant parameters are the same as those in section 4.1.1. But differently, in this case the force $F^{I}(t)$ is assumed to be a non-stationary process with a middle point function $F^{c}(t)=12+2 \sin \omega_{F} t$ and a half width function $F^{r}(t)=2+\sin \omega_{F} t$, as shown in Fig. 13. Two types of self-correlation functions are discussed for $F^{I}(t)$, namely, $r\left(t_{i}, t_{j}\right)=\mathrm{e}^{-\alpha\left|t_{i}-t_{j}\right|} \cos \omega_{F}\left(t_{i}-t_{j}\right)$, and $r\left(t_{i}, t_{j}\right)=0, t_{i} \neq t_{j}$, herein $\alpha=0.2$ and $\omega_{F}$ is a variable parameter. In Fig. 14 (a)-(c), the response bounds of displacement $z(t)$ are computed when $\omega_{F}$ is set as 4,1 and 0.5 , respectively. Because of the periodicity of the middle point function and the half width function, $F^{I}(t)$ also exhibits a variation of periodicity. The obtained response bounds also present two stages: at first they oscillate irregularly in the transient response phase and then both the upper and lower bounds oscillate periodically. Especially, the results show that this periodicity just agrees with that of $F^{I}(t)$. Moreover, from Fig. 14(b) it can be seen that when the frequency $\omega_{F}$ of the force $F^{I}(t)$ equals to the natural frequency $\omega_{n}$ of the system, i.e., when $\omega_{F}=1$, the response bounds for both $r\left(t_{i}, t_{j}\right)=\mathrm{e}^{-\alpha\left|t_{i}-t_{j}\right|} \cos \omega_{F}\left(t_{i}-t_{j}\right)$ and $r\left(t_{i}, t_{j}\right)=0, t_{i} \neq t_{j}$ exhibit strong oscillation characteristics. And interestingly, in this case it seems that the self-correlation function of $F^{I}(t)$ becomes nearly invalid, since the response bounds under $r\left(t_{i}, t_{j}\right)=\mathrm{e}^{-\alpha\left|t_{i}-t_{j}\right|} \cos \omega_{F}\left(t_{i}-t_{j}\right)$ and $r\left(t_{i}, t_{j}\right)=0, t_{i} \neq t_{j}$ are very close. Nevertheless, the results under the dependent process are still bounded by those under the 


\subsection{A 3-DOF system}

3

4

5

7

10

Figure 15 shows a 3-DOF damped system subjected to three external forces $F_{1}(t), F_{2}(t)$ and $F_{3}(t)$. The equation of motion can be expressed as Eq. (31) with,

$$
\mathbf{M}=\left[\begin{array}{ccc}
m_{1} & 0 & 0 \\
0 & m_{2} & 0 \\
0 & 0 & m_{3}
\end{array}\right], \mathbf{C}=\left[\begin{array}{ccc}
c_{1}+c_{2}+c_{3} & -c_{2} & -c_{5} \\
-c_{2} & c_{2}+c_{3}+c_{4} & -c_{3} \\
-c_{5} & -c_{3} & c_{3}+c_{5}
\end{array}\right], \mathbf{K}=\left[\begin{array}{ccc}
k_{1}+k_{2} & -k_{2} & 0 \\
-k_{2} & k_{2}+k_{3} & -k_{3} \\
0 & -k_{3} & k_{3}
\end{array}\right], \mathbf{F}=\left[\begin{array}{c}
F_{1}(t) \\
F_{2}(t) \\
F_{3}(t)
\end{array}\right]
$$

where

$$
\begin{aligned}
& m_{1}=m_{2}=m_{3}=1 \\
& c_{1}=c_{2}=c_{3}=1, \quad c_{4}=c_{5}=0.3 \\
& k_{1}=k_{2}=k_{3}=1
\end{aligned}
$$

8 The external forces are all treated as stationary interval processes and independent with each

9 other. Their middle point functions and half width functions are as follows,

10

$$
\begin{aligned}
& F_{1}^{c}(t)=3, F_{2}^{c}(t)=5, F_{3}^{c}(t)=10 \\
& F_{1}^{r}(t)=1.8, F_{2}^{r}(t)=3.0, F_{3}^{r}(t)=4.8
\end{aligned}
$$

The time step $\Delta t$ is set as $0.5 \mathrm{~s}$.

A same self-correlation function is set to the three forces $F_{1}(t), F_{2}(t)$ and $F_{3}(t)$, and two different cases for the self-correlation function are considered, namely $r_{F}(\tau)=\mathrm{e}^{-\alpha|\tau|}, \alpha=0.2$ and $r_{F}(\tau)=0$. Corresponding displacement responses of the three mass blocks are calculated and plotted in Fig. 16, from which two similar phenomena as the SDOF case could be found. Firstly, the response bounds of $r_{F}(\tau)=\mathrm{e}^{-\alpha|\tau|}$ are enveloped by those of the independent process case $\left(r_{F}(\tau)=0\right)$. Secondly, for this damped system, both the upper and lower bounds of the displacements tend to be steady after an initial phase. The displacement intervals at the steady state for $r_{F}(\tau)=\mathrm{e}^{-\alpha|\tau|}$ are $z_{1}^{I}=[8.40,27.59]$, 
$1 z_{2}^{I}=[15.60,50.40]$ and $z_{3}^{I}=[20.80,65.20]$, respectively. Corresponding results for

$2 \quad r_{F}(\tau)=0$ are $z_{1}^{I}=[4.28,31.72], \quad z_{2}^{I}=[8.01,57.99]$ and $z_{3}^{I}=[11.09,74.91]$, respectively,

3 which are obviously wider than those for $r_{F}(\tau)=\mathrm{e}^{-\alpha|\tau|}$.

4 In addition, an undamped case is also investigated for the above 3-DOF system with

$5 c_{i}, i=1,2, \ldots, 5$ equal to 0 and other parameters unchanged. Through non-random vibration

6 analysis, the displacement responses are obtained and plotted in Fig. 17. The results show that

7 for the umdamped system, a steady state of the dynamic responses is not reached just as we

8 predict. Besides, since no energy dissipating damping exists for the system, the dynamic

9 response bounds expand gradually with time, which is also consistent with the actual situations. In addition, the result illustrates again that the response bounds for dependent

11 processes are enveloped by those for independent process.

\subsection{A practical half vehicle vibration problem}

Vehicle vibration and dynamic analysis have been a hot research topic due to its important role in ride comfort and overall vehicle performance. When the vehicle rides over a rough road, the vibration of the vehicle body can make passengers feel uncomfortable, and even cause damages to vehicle components. Therefore, to ensure the ride comfort and reliability of vehicle parts, it is vital to have a good knowledge on the vibration behavior of the vehicle that rides on rough road surfaces. In this section, the proposed non-random vibration method is tried for application in vehicle vibration analysis. A simplified 5-DOF model of vehicle [58] under road excitations is considered as shown in Fig. 18. The vehicle velocity is set to $v=20 \mathrm{~m} / \mathrm{s}$. Other constant parameters of the model are listed in Table 2. The equation of 
motion can be expressed as [58],

$$
\mathbf{M Z}+\mathbf{C} \dot{\mathbf{Z}}+\mathbf{K Z}=\mathbf{K}_{t} \mathbf{Q}
$$

3 where

5

$\mathbf{M}=\left[\begin{array}{ccccc}m_{s} & 0 & 0 & 0 & 0 \\ 0 & m_{3} & 0 & 0 & 0 \\ 0 & 0 & I_{x} & 0 & 0 \\ 0 & 0 & 0 & m_{1} & 0 \\ 0 & 0 & 0 & 0 & m_{2}\end{array}\right], \quad \mathbf{C}=\left[\begin{array}{ccccc}c_{s} & -c_{s} & L c_{s} & 0 & 0 \\ -c_{s} & c_{s}+c_{3}+c_{4} & -L c_{s}-a c_{3}+b c_{4} & -c_{3} & -c_{4} \\ L c_{s} & -L c_{s}-a c_{3}+b c_{4} & L^{2} c_{s}+a^{2} c_{3}+b^{2} c_{4} & a c_{3} & -b c_{4} \\ 0 & -c_{3} & a c_{3} & c_{3} & 0 \\ 0 & -c_{4} & -b c_{4} & 0 & c_{4}\end{array}\right]$

6

$$
\mathbf{K}=\left[\begin{array}{ccccc}
k_{s} & -k_{s} & L k_{s} & 0 & 0 \\
-k_{s} & k_{s}+k_{3}+k_{4} & -L k_{s}-a k_{3}+b k_{4} & -k_{3} & -k_{4} \\
L k_{s} & -L k_{s}-a k_{3}+b k_{4} & L^{2} k_{s}+a^{2} k_{3}+b^{2} k_{4} & a k_{3} & -b k_{4} \\
0 & -k_{3} & a k_{3} & k_{1}+k_{3} & 0 \\
0 & -k_{4} & -b k_{4} & 0 & k_{2}+k_{4}
\end{array}\right], \quad \mathbf{K}_{t}=\left[\begin{array}{cc}
0 & 0 \\
0 & 0 \\
0 & 0 \\
k_{1} & 0 \\
0 & k_{2}
\end{array}\right]
$$

$$
\mathbf{Z}=\left[\begin{array}{c}
z_{s}(t) \\
z_{3}(t) \\
\theta(t) \\
z_{1}(t) \\
z_{2}(t)
\end{array}\right], \mathbf{Q}=\left[\begin{array}{l}
Q_{1}(t) \\
Q_{2}(t)
\end{array}\right]
$$

$7 \mathbf{Z}, \dot{\mathbf{Z}}, \ddot{\mathbf{Z}}$ are displacement, velocity and acceleration vectors, respectively; $\mathbf{Q}$ is the road

8 excitation vector. Herein the excitations $Q_{1}(t)$ and $Q_{2}(t)$ are modeled as stationary interval

9 processes with upper bound functions $Q_{1}^{U}(t)=Q_{2}^{U}(t)=0.05 \mathrm{~m}$ and lower bound functions $Q_{1}^{L}(t)=Q_{2}^{L}(t)=-0.05 \mathrm{~m}$. The self-correlation functions of $Q_{1}(t)$ and $Q_{2}(t)$ are $r_{1}(\tau)=r_{2}(\tau)=\mathrm{e}^{-\alpha|\tau|}, \alpha=0.01$. The time step $\Delta t$ is set as $0.03 \mathrm{~s}$.

The excitations $Q_{1}(t)$ and $Q_{2}(t)$ are firstly treated as independent with each other, namely, for $\forall t_{i}, t_{j}$, there is $r\left(Q_{1}\left(t_{i}\right), Q_{2}\left(t_{j}\right)\right)=0$. The bounds of the possible responses of $z_{s}(t), z_{1}(t), z_{2}(t), z_{3}(t)$ and $\theta(t)$ are calculated and plotted in Fig. 19, from which it can be found that the response bounds all tend to be steady with time. The maximum response intervals in the given period and the response intervals at the steady state are both given in 
obviously narrower than the corresponding maximum response intervals.

Practically, since the rear tire moves at the same road profile as forward tire with a time delay of $\Delta t=\frac{a+b}{v}$, i.e., $Q_{1}(t)=Q_{2}(t+\Delta t)$, the mutual correlation between the uncertain excitations $Q_{1}(t)$ and $Q_{2}(t)$ should be considered. Therefore there is,

$$
r\left(Q_{1}\left(t_{i}\right), Q_{2}\left(t_{i}+\tau\right)\right)=r\left(Q_{1}\left(t_{i}\right), Q_{1}\left(t_{i}+\tau-\Delta t\right)\right)=r(\tau-\Delta t)
$$

Namely, in this case the mutual-correlation function between $Q_{1}(t)$ at time $t_{i}$ and $Q_{2}(t)$ at $t_{j}=t_{i}+\tau$ can be calculated by the self-correlation function of $Q_{1}(t)$ with time interval $\tau-\Delta t$. Based on this, corresponding results are computed as shown in Fig. 20, and the response bounds at the steady state are listed in Table 4. By comparing Fig. 20 with Fig. 19, obvious difference of the responses of the pitch angle $\theta$ can be found when the mutual-correlation of the road excitations is considered. From the third sub graph of Fig. 20 we can see that the vehicle tends to run steadily with pitch angle constant to almost $0^{\circ}$, which means the "nodding" phenomenon can hardly exist with the given speed driving on this kind of road.

By applying the non-random vibration method to the practical vehicle vibration analysis, the upper and lower bounds of dynamic responses can be easily obtained, which are very useful for analysis and design of the suspended system of vehicle. However, it should be pointed out that since only limited information is required in our interval process compared with the traditional stochastic process, some more detailed characteristics may not be conveyed in the response analysis results. Take the results shown in Fig. 20 as an example, we can find that the response bounds for displacement of the chair $z_{s}(t)$ and those of the vehicle body $z_{3}(t)$ are similar. Nevertheless, it is known that for the driver's 
comfortableness the chair generally vibrates more smoothly than the vehicle body. For instance, if we designate $Q_{1}(t)=0.05 \cos \omega(t+\Delta t)$ and $Q_{2}(t)=0.05 \cos \omega t \quad(\omega=20 \pi)$ as deterministic input excitations, it can be found from Fig. 21 that the displacement of the chair $z_{s}(t)$ exhibits a more stable variation than that of the vehicle body. However, such details cannot be reflected in the non-random vibration analysis results.

\section{Conclusions}

In this paper, an interval process model for time-varying or dynamic uncertainty quantification is proposed, in which only the upper and lower bounds of the uncertain parameter are required rather than its precise probability distribution. Some basic concepts and definitions are given to better describe the interval process model. A matrix-decomposition-based method is presented to transform the dependent interval process into an independent one, which greatly facilitates the subsequent uncertainty analysis. More importantly, by introducing the interval process model, a non-random vibration analysis method is proposed to compute the dynamic response bounds of a vibration system subjected to uncertain excitations. In the numerical examples, various cases of the vibration systems and uncertain inputs are considered, and three main phenomena are observed from the results. Firstly, by using the non-random vibration analysis, the dynamic responses can be given in the form of upper and lower bounds which envelop all possible time-history responses under the time-varying uncertain excitations. Secondly, the results for SDOF and MDOF systems all exhibit reasonable dynamic characteristics, which are consistent with actual physical situations. Specifically, for damped systems, the dynamic response bounds experience two 
1 stages, namely the transient response stage and the steady-state response stage; while for

2 undamped systems, the bounds expand continuously with time, and hence a steady state

3 cannot be reached. Thirdly, different kinds of self-correlation functions of the input

4 excitations can have significant impacts on the response bounds of the vibration systems.

5 Nevertheless, the response bounds under the dependent process are always enveloped by

6 those under the independent process. With such property, an independent interval process can

7 always be applied to obtain a relatively wide response bounds when the self-correlation

8 function is difficult to create, thus guaranteeing a safety analysis result for practical reliability

9 design.

\section{Acknowledgements}

This work is supported by the National Science Foundation of China (11172096), the

\section{Appendix}

\section{Appendix A}

Proof. Denote the sample matrix $\mathbf{x}$ and the middle point matrix $\mathbf{X}^{C}$ as, 


$$
\begin{aligned}
\mathbf{X} & =\left[\begin{array}{cccc}
x_{1}^{(1)} & x_{1}^{(2)} & \ldots & x_{1}^{(m)} \\
x_{2}^{(1)} & x_{2}^{(2)} & \ldots & x_{2}^{(m)} \\
\vdots & \vdots & \vdots & \vdots \\
x_{n}^{(1)} & x_{n}^{(2)} & \ldots & x_{n}^{(m)}
\end{array}\right]_{n \times m} \\
\mathbf{X}^{C} & =\left[\begin{array}{cccc}
X_{1}^{c} & X_{1}^{c} & \ldots & X_{1}^{c} \\
X_{2}^{c} & X_{2}^{c} & \ldots & X_{2}^{c} \\
\vdots & \vdots & \vdots & \vdots \\
X_{n}^{c} & X_{n}^{c} & \ldots & X_{n}^{c}
\end{array}\right]_{n \times m}
\end{aligned}
$$

2 where $x_{i}^{(p)}$ is the value of the $p^{\text {th }}$ sample function of the interval process $X^{I}(t)$ at time $t_{i}$, 3 and $X_{i}^{c}=X^{c}\left(t_{i}\right)$ is the value of its middle point function at time $t_{i}$. From the definition of

4 self-correlation function in Eq. (5), the correlation matrix can be rewritten in the following

5 form,

$$
\mathbf{R}_{X}=\mathbf{W}_{X}^{-1}\left[\frac{1}{m}\left(\mathbf{x}-\mathbf{X}^{C}\right)\left(\mathbf{x}-\mathbf{X}^{C}\right)^{\mathrm{T}}\right] \mathbf{W}_{X}^{-1}
$$

7 Denote $\mathbf{Y}^{I}=\mathbf{L}^{-1} \mathbf{X}^{I}$ as the vector form of the independent interval process $Y^{I}(t)$ that 8 transformed from $X^{I}(t)$, where $\mathbf{L}=\mathbf{W}_{X} \mathbf{L}_{X}$. Correspondingly, its sample matrix $\mathbf{y}$ can be 9 obtained as,

$$
\mathbf{y}=\mathbf{L}^{-1} \mathbf{x}
$$

As proved in Appendix $\mathbf{B}$, there is,

$$
\mathbf{Y}^{c}=\mathbf{L}^{-1} \mathbf{X}^{c}
$$

For the convenience of expression, denote $\boldsymbol{\Gamma}$ as the matrix $\mathbf{L}^{-1}$ and $\boldsymbol{\Gamma}_{i}$ as the $i^{\text {th }}$ row of $\mathbf{L}^{-1}, \quad i=1,2, \ldots, n$. Hence Eqs. (A.3) and (A.4) can be represented by,

$$
\mathbf{y}=\Gamma \mathbf{x}
$$

$$
\mathbf{Y}^{c}=\boldsymbol{\Gamma} \mathbf{X}^{c}
$$

17 Then the entries $W_{i}^{Y}$ of the diagonal matrix $\mathbf{W}_{Y}$ can be calculated as, 


$$
\begin{aligned}
W_{i}^{Y} & =\sqrt{\frac{1}{m} \sum_{p=1}^{m}\left(y_{i}^{(p)}-Y_{i}^{c}\right)^{2}} \\
& =\sqrt{\frac{1}{m}\left[\boldsymbol{\Gamma}_{i}\left(\mathbf{x}-\mathbf{X}^{C}\right)\right]\left[\boldsymbol{\Gamma}_{i}\left(\mathbf{x}-\mathbf{X}^{C}\right)\right]^{\mathrm{T}}} \\
& =\sqrt{\boldsymbol{\Gamma}_{i}\left[\frac{1}{m}\left(\mathbf{x}-\mathbf{X}^{C}\right)\left(\mathbf{x}-\mathbf{X}^{C}\right)^{\mathrm{T}}\right] \boldsymbol{\Gamma}_{i}^{\mathrm{T}}}
\end{aligned}
$$

2 From Eq. (A.2), there is,

3

$$
\frac{1}{m}\left(\mathbf{x}-\mathbf{X}^{C}\right)\left(\mathbf{x}-\mathbf{X}^{C}\right)^{\mathrm{T}}=\mathbf{W}_{X} \mathbf{R}_{X} \mathbf{W}_{X}^{\mathrm{T}}
$$

4 By substituting Eq. (A.8) into Eq. (A.7) yields,

5

$$
\begin{aligned}
W_{i}^{Y} & =\sqrt{\boldsymbol{\Gamma}_{i}\left[\frac{1}{m}\left(\mathbf{x}-\mathbf{X}^{C}\right)\left(\mathbf{x}-\mathbf{X}^{C}\right)^{\mathrm{T}}\right] \boldsymbol{\Gamma}_{i}^{\mathrm{T}}} \\
& =\sqrt{\boldsymbol{\Gamma}_{i} \mathbf{W}_{X} \mathbf{R}_{X} \mathbf{W}_{X}^{\mathrm{T}} \boldsymbol{\Gamma}_{i}^{\mathrm{T}}} \\
& =\sqrt{\boldsymbol{\Gamma}_{i} \mathbf{W}_{X} \mathbf{L}_{X} \mathbf{L}_{X}^{\mathrm{T}} \mathbf{W}_{X}^{\mathrm{T}} \boldsymbol{\Gamma}_{i}^{\mathrm{T}}} \\
& =\sqrt{\boldsymbol{\Gamma}_{i} \mathbf{L} \mathbf{L}^{\mathrm{T}} \boldsymbol{\Gamma}_{i}^{\mathrm{T}}} \\
& =1
\end{aligned}
$$

Subsequently, the correlation matrix $\mathbf{R}_{Y}$ of $\mathbf{Y}^{I}$ can be obtained,

$$
\begin{aligned}
\mathbf{R}_{Y} & =\mathbf{W}_{Y}^{-1}\left[\frac{1}{m}\left(\mathbf{y}-\mathbf{Y}^{C}\right)\left(\mathbf{y}-\mathbf{Y}^{C}\right)^{\mathrm{T}}\right] \mathbf{W}_{Y}^{-1} \\
& =\frac{1}{m}\left(\mathbf{y}-\mathbf{Y}^{C}\right)\left(\mathbf{y}-\mathbf{Y}^{C}\right)^{\mathrm{T}} \\
& =\frac{1}{m}\left(\mathbf{L}^{-1}\left(\mathbf{x}-\mathbf{X}^{C}\right)\right)\left(\mathbf{L}^{-1}\left(\mathbf{x}-\mathbf{X}^{C}\right)\right)^{\mathrm{T}} \\
& =\mathbf{L}^{-1}\left[\frac{1}{m}\left(\mathbf{x}-\mathbf{X}^{C}\right)\left(\mathbf{x}-\mathbf{X}^{C}\right)^{\mathrm{T}}\right]\left(\mathbf{L}^{-1}\right)^{\mathrm{T}} \\
& =\mathbf{L}^{-1} \mathbf{W}_{X} \mathbf{R} \mathbf{W}_{X}^{\mathrm{T}}\left(\mathbf{L}^{-1}\right)^{\mathrm{T}} \\
& =\mathbf{L}^{-1} \mathbf{W}_{X} \mathbf{L}_{X} \mathbf{L}_{X}^{\mathrm{T}} \mathbf{W}_{X}^{\mathrm{T}}\left(\mathbf{L}^{-1}\right)^{\mathrm{T}} \\
& =\mathbf{I}
\end{aligned}
$$

$8 \quad$ Herein I stands for the identity matrix.

9 Q. E. D.

\section{Appendix B}

Proof. Rewrite the transformation $\mathbf{Y}^{I}=\mathbf{L}^{-1} \mathbf{X}^{I}$ in the form of $\mathbf{X}^{I}=\mathbf{L} \mathbf{Y}^{I}$, 


$$
\left[\begin{array}{c}
{\left[X_{1}^{L}, X_{1}^{U}\right]} \\
{\left[X_{2}^{L}, X_{2}^{U}\right]} \\
\vdots \\
{\left[X_{n}^{L}, X_{n}^{U}\right]}
\end{array}\right]=\mathbf{L}\left[\begin{array}{c}
{\left[Y_{1}^{L}, Y_{1}^{U}\right]} \\
{\left[Y_{2}^{L}, Y_{2}^{U}\right]} \\
\vdots \\
{\left[Y_{n}^{L}, Y_{n}^{U}\right]}
\end{array}\right]=\left[\begin{array}{cccc}
l_{11} & l_{12} & \ldots & l_{1 n} \\
l_{21} & l_{22} & \ldots & l_{2 n} \\
\vdots & \vdots & \ddots & \vdots \\
l_{n 1} & l_{n 2} & \ldots & l_{n n}
\end{array}\right]\left[\begin{array}{c}
{\left[Y_{1}^{L}, Y_{1}^{U}\right]} \\
{\left[Y_{2}^{L}, Y_{2}^{U}\right]} \\
\vdots \\
{\left[Y_{n}^{L}, Y_{n}^{U}\right]}
\end{array}\right]
$$

2 where $l_{i j}$ means the entry in the $i^{\text {th }}$ row and $j^{\text {th }}$ column of the matrix $\mathbf{L}$; and for $j>i$ there is

$3 \quad l_{i j}=0$. Specifically,

4

$$
\begin{aligned}
X_{i}^{I} & =\left[X_{i}^{L}, X_{i}^{U}\right] \\
& =l_{i 1} \cdot\left[Y_{1}^{L}, Y_{1}^{U}\right]+l_{i 2} \cdot\left[Y_{2}^{L}, Y_{2}^{U}\right]+\ldots+l_{i n} \cdot\left[Y_{n}^{L}, Y_{n}^{U}\right] \\
& =l_{i 1} \cdot\left(Y_{1}^{c}+Y_{1}^{r} \cdot[-1,1]\right)+l_{i 2} \cdot\left(Y_{2}^{c}+Y_{2}^{r} \cdot[-1,1]\right)+\ldots+l_{i n} \cdot\left(Y_{n}^{c}+Y_{n}^{r} \cdot[-1,1]\right) \\
& =\left(l_{i 1} Y_{1}^{c}+l_{i 2} Y_{2}^{c}+\ldots+l_{i n} Y_{n}^{c}\right)+\left(l_{i 1} Y_{1}^{r} \cdot[-1,1]+l_{i 2} Y_{2}^{r} \cdot[-1,1]+\ldots+l_{i n} Y_{n}^{r} \cdot[-1,1]\right)
\end{aligned}
$$

5

namely,

$$
\begin{aligned}
& {\left[X_{i}^{c}-X_{i}^{r}, X_{i}^{c}+X_{i}^{r}\right]} \\
& =\left(l_{i 1} Y_{1}^{c}+l_{i 2} Y_{2}^{c}+\ldots+l_{i n} Y_{n}^{c}\right)+\left(l_{i 1} Y_{1}^{r} \cdot[-1,1]+l_{i 2} Y_{2}^{r} \cdot[-1,1]+\ldots+l_{i n} Y_{n}^{r} \cdot[-1,1]\right) \\
& =\left(\sum_{k=1}^{n} l_{i k} Y_{k}^{c}\right)+\left(\sum_{k=1}^{n}\left|l_{i k} Y_{k}^{r}\right|\right) \cdot[-1,1] \\
& =\left[\left(\sum_{k=1}^{n} l_{i k} Y_{k}^{c}-\sum_{k=1}^{n}\left|l_{i k} Y_{k}^{r}\right|\right),\left(\sum_{k=1}^{n} l_{i k} Y_{k}^{c}+\sum_{k=1}^{n}\left|l_{i k} Y_{k}^{r}\right|\right)\right]
\end{aligned}
$$

7 It is not difficult to obtain that,

8

$$
X_{i}^{c}=\sum_{k=1}^{n} l_{i k} Y_{k}^{c}
$$

$$
X_{i}^{r}=\sum_{k=1}^{n}\left|l_{i k} Y_{k}^{r}\right|=\sum_{k=1}^{n}\left|l_{i k}\right| Y_{k}^{r}
$$

9

namely,

10

$$
\left[\begin{array}{c}
X_{1}^{c} \\
X_{2}^{c} \\
\vdots \\
X_{n}^{c}
\end{array}\right]=\left[\begin{array}{cccc}
l_{11} & l_{12} & \ldots & l_{1 n} \\
l_{21} & l_{22} & \ldots & l_{2 n} \\
\vdots & \vdots & \ddots & \vdots \\
l_{n 1} & l_{n 2} & \ldots & l_{n n}
\end{array}\right]\left[\begin{array}{c}
Y_{1}^{c} \\
Y_{2}^{c} \\
\vdots \\
Y_{n}^{c}
\end{array}\right]
$$

$$
\left[\begin{array}{c}
X_{1}^{r} \\
X_{2}^{r} \\
\vdots \\
X_{n}^{r}
\end{array}\right]=\left[\begin{array}{cccc}
\left|l_{11}\right| & \left|l_{12}\right| & \ldots & \left|l_{1 n}\right| \\
\left|l_{21}\right| & \left|l_{22}\right| & \ldots & \left|l_{2 n}\right| \\
\vdots & \vdots & \ddots & \vdots \\
\left|l_{n 1}\right| & \left|l_{n 2}\right| & \ldots & \left|l_{n n}\right|
\end{array}\right]\left[\begin{array}{c}
Y_{1}^{r} \\
Y_{2}^{r} \\
\vdots \\
Y_{n}^{r}
\end{array}\right]
$$

Denote, 


$$
\begin{aligned}
& \mathbf{X}^{c}=\left[\begin{array}{llll}
X_{1}^{c} & X_{2}^{c} & \ldots & X_{n}^{c}
\end{array}\right]^{\mathrm{T}}, \quad \mathbf{X}^{r}=\left[\begin{array}{llll}
X_{1}^{r} & X_{2}^{r} & \ldots & X_{n}^{r}
\end{array}\right]^{\mathrm{T}} \\
& \mathbf{Y}^{c}=\left[\begin{array}{llll}
Y_{1}^{c} & Y_{2}^{c} & \ldots & Y_{n}^{c}
\end{array}\right]^{\mathrm{T}}, \quad \mathbf{Y}^{r}=\left[\begin{array}{llll}
Y_{1}^{r} & Y_{2}^{r} & \ldots & Y_{n}^{r}
\end{array}\right]^{\mathrm{T}} \\
& {[|\mathbf{L}|]=\left[\begin{array}{cccc}
\left|l_{11}\right| & \left|l_{12}\right| & \ldots & \left|l_{1 n}\right| \\
\left|l_{21}\right| & \left|l_{22}\right| & \ldots & \left|l_{2 n}\right| \\
\vdots & \vdots & \ddots & \vdots \\
\left|l_{n 1}\right| & \left|l_{n 2}\right| & \ldots & \left|l_{n n}\right|
\end{array}\right]}
\end{aligned}
$$

2 Then Eq. (B.4) or Eq. (B.5) can be rewritten in the following form,

3

$$
\begin{aligned}
& \mathbf{X}^{c}=\mathbf{L} \mathbf{Y}^{c} \\
& \mathbf{X}^{r}=[|\mathbf{L}|] \mathbf{Y}^{r}
\end{aligned}
$$

4 Since that the diagonal entries of the lower triangular matrix $\mathbf{L}$ are all nonzero, both $\mathbf{L}$ and $5[|\mathbf{L}|]$ are invertible, and hence there is,

6

$$
\begin{aligned}
\mathbf{Y}^{c} & =\mathbf{L}^{-1} \mathbf{X}^{c} \\
\mathbf{Y}^{r} & =[|\mathbf{L}|]^{-1} \mathbf{X}^{r}
\end{aligned}
$$

7 Q. E. D.

8

\section{Appendix C}

Rewrite Eq. (19) in the form of matrix equality,

11

$$
\left[\begin{array}{c}
z_{j} \\
\dot{z}_{j}
\end{array}\right]=\left[\begin{array}{cccc}
A_{j} & B_{j}-A_{j} & C_{j} & D_{j} \\
\bar{A}_{j} & \bar{B}_{j}-\bar{A}_{j} & \bar{C}_{j} & \bar{D}_{j}
\end{array}\right]\left[\begin{array}{c}
F_{j} \\
F_{j-1} \\
z_{j-1} \\
\dot{z}_{j-1}
\end{array}\right]
$$

12

13
By utilizing Eq. (19) or Eq. (C.1) repeatedly, the following recurrence sequences can be obtained, 


$$
\begin{aligned}
& {\left[\begin{array}{c}
F_{j} \\
F_{j-1} \\
z_{j-1} \\
\dot{z}_{j-1}
\end{array}\right]=\left[\begin{array}{ccccc}
1 & 0 & 0 & 0 & 0 \\
0 & 1 & 0 & 0 & 0 \\
0 & A_{j-1} & B_{j-1}-A_{j-1} & C_{j-1} & D_{j-1} \\
0 & \bar{A}_{j-1} & \bar{B}_{j-1}-\bar{A}_{j-1} & \bar{C}_{j-1} & \bar{D}_{j-1}
\end{array}\right]\left[\begin{array}{c}
F_{j} \\
F_{j-1} \\
F_{j-2} \\
z_{j-2} \\
\dot{z}_{j-2}
\end{array}\right]=\mathbf{H}_{j-1}\left[\begin{array}{c}
F_{j} \\
F_{j-1} \\
F_{j-2} \\
z_{j-2} \\
\dot{z}_{j-2}
\end{array}\right]} \\
& {\left[\begin{array}{c}
F_{j} \\
F_{j-1} \\
F_{j-2} \\
z_{j-2} \\
\dot{z}_{j-2}
\end{array}\right]=\left[\begin{array}{cccccc}
1 & 0 & 0 & 0 & 0 & 0 \\
0 & 1 & 0 & 0 & 0 & 0 \\
0 & 0 & 1 & 0 & 0 & 0 \\
0 & 0 & A_{j-2} & B_{j-2}-A_{j-2} & C_{j-2} & D_{j-2} \\
0 & 0 & \bar{A}_{j-2} & \bar{B}_{j-2}-\bar{A}_{j-2} & \bar{C}_{j-2} & \bar{D}_{j-2}
\end{array}\right]\left[\begin{array}{c}
F_{j} \\
F_{j-1} \\
F_{j-2} \\
F_{j-3} \\
z_{j-3} \\
\dot{z}_{j-3}
\end{array}\right]=\mathbf{H}_{j-2}\left[\begin{array}{c}
F_{j} \\
F_{j-1} \\
F_{j-2} \\
F_{j-3} \\
z_{j-3} \\
\dot{z}_{j-3}
\end{array}\right]} \\
& {\left[\begin{array}{c}
F_{j} \\
F_{j-1} \\
\vdots \\
F_{1} \\
z_{1} \\
\dot{z}_{1}
\end{array}\right]=\left[\begin{array}{ccccccc}
1 & 0 & \ldots & 0 & 0 & 0 & 0 \\
0 & 1 & \ldots & 0 & 0 & 0 & 0 \\
\vdots & \vdots & \ddots & 0 & 0 & 0 & 0 \\
0 & 0 & 0 & 1 & 0 & 0 & 0 \\
0 & 0 & 0 & A_{1} & B_{1}-A_{1} & C_{1} & D_{1} \\
0 & 0 & 0 & \bar{A}_{1} & \bar{B}_{1}-\bar{A}_{1} & \bar{C}_{1} & \bar{D}_{1}
\end{array}\right]\left[\begin{array}{c}
F_{j} \\
F_{j-1} \\
\vdots \\
F_{1} \\
F_{0} \\
z_{0} \\
\dot{z}_{0}
\end{array}\right]=\mathbf{H}_{1}\left[\begin{array}{c}
F_{j} \\
F_{j-1} \\
\vdots \\
F_{1} \\
F_{0} \\
z_{0} \\
\dot{z}_{0}
\end{array}\right]}
\end{aligned}
$$

2 Hence there is,

3

$$
\left[\begin{array}{c}
F_{j} \\
F_{j-1} \\
z_{j-1} \\
\dot{z}_{j-1}
\end{array}\right]=\mathbf{H}_{j-1} \mathbf{H}_{j-2} \ldots \mathbf{H}_{1}\left[\begin{array}{c}
F_{j} \\
F_{j-1} \\
\vdots \\
F_{1} \\
F_{0} \\
z_{0} \\
\dot{z}_{0}
\end{array}\right]
$$

$4 \quad$ Let $\mathbf{H}^{*}=\left[\begin{array}{cccc}A_{j} & B_{j}-A_{j} & C_{j} & D_{j} \\ \bar{A}_{j} & \bar{B}_{j}-\bar{A}_{j} & \bar{C}_{j} & \bar{D}_{j}\end{array}\right], \quad \mathbf{H}=\mathbf{H}^{*} \prod_{k=1}^{j-1} \mathbf{H}_{k}, \quad \mathbf{F}=\left[\begin{array}{lllll}F_{j} & F_{j-1} & \ldots & F_{1} & F_{0}\end{array}\right]^{\mathrm{T}}, \quad$ and $5 \quad \mathbf{z}_{0}=\left[\begin{array}{ll}z_{0} & \dot{z}_{0}\end{array}\right]^{\mathrm{T}}$, then Eq. (C.1) can be expressed as,

6

$$
\left[\begin{array}{c}
z_{j} \\
\dot{z}_{j}
\end{array}\right]=\mathbf{H}\left[\begin{array}{c}
\mathbf{F} \\
\mathbf{z}_{0}
\end{array}\right]
$$

7

\section{Appendix D}

9 From Eq. (41) there is, 


$$
\begin{aligned}
& \mathbf{Z}\left(t_{j+1}\right)=\hat{\mathbf{M}}^{-1} \hat{\mathbf{F}}\left(t_{j}\right) \\
& =\hat{\mathbf{M}}^{-1}\left(\mathbf{F}\left(t_{j}\right)-\left(\mathbf{K}-a_{2} \mathbf{M}\right) \mathbf{Z}\left(t_{j}\right)-\left(a_{0} \mathbf{M}-a_{1} \mathbf{C}\right) \mathbf{Z}\left(t_{j-1}\right)\right) \\
& =\left[\begin{array}{lll}
\hat{\mathbf{M}}^{-1} & -\hat{\mathbf{M}}^{-1}\left(\mathbf{K}-a_{2} \mathbf{M}\right) & -\hat{\mathbf{M}}^{-1}\left(a_{0} \mathbf{M}-a_{1} \mathbf{C}\right)
\end{array}\right]\left[\begin{array}{c}
\mathbf{F}\left(t_{j}\right) \\
\mathbf{Z}\left(t_{j}\right) \\
\mathbf{Z}\left(t_{j-1}\right)
\end{array}\right] \\
& =\hat{\mathbf{H}}^{*}\left[\begin{array}{c}
\mathbf{F}\left(t_{j}\right) \\
\mathbf{Z}\left(t_{j}\right) \\
\mathbf{Z}\left(t_{j-1}\right)
\end{array}\right]
\end{aligned}
$$

2 From Eq. (40) and Eq. (41) it can be obtained that,

$$
\begin{aligned}
& {\left[\begin{array}{c}
\mathbf{F}\left(t_{j}\right) \\
\mathbf{Z}\left(t_{j}\right) \\
\mathbf{Z}\left(t_{j-1}\right)
\end{array}\right]=\left[\begin{array}{c:c:c:c}
\mathbf{I} & \mathbf{O} & \mathbf{0} & \mathbf{0} \\
\hdashline \mathbf{O} & \hat{\mathbf{M}}^{-1} & -\hat{\mathbf{M}}^{-1}\left(\mathbf{K}-a_{2} \mathbf{M}\right) & -\hat{\mathbf{M}}^{-1}\left(a_{0} \mathbf{M}-a_{1} \mathbf{C}\right) \\
\hdashline \mathbf{O} & \mathbf{O} & \mathbf{I} & \mathbf{O}
\end{array}\right]\left[\begin{array}{c}
\mathbf{F}\left(t_{j}\right) \\
\mathbf{F}\left(t_{j-1}\right) \\
\mathbf{Z}\left(t_{j-1}\right) \\
\mathbf{Z}\left(t_{j-2}\right)
\end{array}\right]=\hat{\mathbf{H}_{1}}\left[\begin{array}{c}
\mathbf{F}\left(t_{j}\right) \\
\mathbf{F}\left(t_{j-1}\right) \\
\mathbf{Z}\left(t_{j-1}\right) \\
\mathbf{Z}\left(t_{j-2}\right)
\end{array}\right]} \\
& {\left[\begin{array}{c}
\mathbf{F}\left(t_{j}\right) \\
\mathbf{F}\left(t_{j-1}\right) \\
\mathbf{Z}\left(t_{j-1}\right) \\
\mathbf{Z}\left(t_{j-2}\right)
\end{array}\right]=\left[\begin{array}{c:c:c:c:c}
\mathbf{I} & \mathbf{O} & \ldots & \ldots & \mathbf{O} \\
\hdashline \mathbf{O} & \mathbf{I} & \mathbf{O} & \cdots & \mathbf{O} \\
\hdashline \vdots & \ddots & \hat{\mathbf{M}}^{-1} & -\hat{\mathbf{M}}^{-1}\left(\mathbf{K}-a_{2} \mathbf{M}\right) & -\hat{\mathbf{M}}^{-1}\left(a_{0} \mathbf{M}-a_{1} \mathbf{C}\right) \\
\hdashline \mathbf{O} & \cdots & \mathbf{O} & \mathbf{I} & \mathbf{O}
\end{array}\right]\left[\begin{array}{c}
\mathbf{F}\left(t_{j}\right) \\
\mathbf{F}\left(t_{j-1}\right) \\
\mathbf{F}\left(t_{j-2}\right) \\
\mathbf{Z}\left(t_{j-2}\right) \\
\mathbf{Z}\left(t_{j-3}\right)
\end{array}\right]=\hat{\mathbf{H}_{2}}\left[\begin{array}{c}
\mathbf{F}\left(t_{j}\right) \\
\mathbf{F}\left(t_{j-1}\right) \\
\mathbf{F}\left(t_{j-2}\right) \\
\mathbf{Z}\left(t_{j-2}\right) \\
\mathbf{Z}\left(t_{j-3}\right)
\end{array}\right]}
\end{aligned}
$$

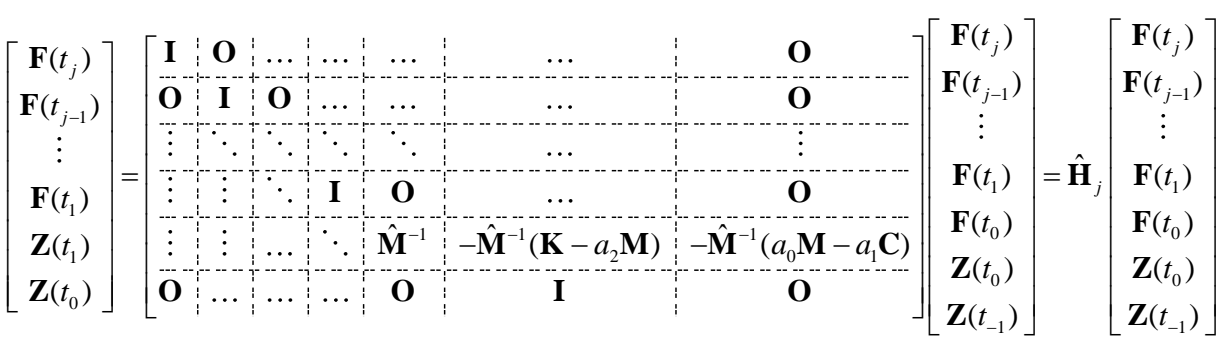

\begin{tabular}{|c|c|c|c|c|c|c|c|c|c|c|c|}
\hline $\mathbf{F}\left(t_{j}\right)$ & 1 & C & & & & & ... & $\mathbf{O}$ & {$\left[\mathbf{F}\left(t_{j}\right)\right]$} & & $\mathbf{F}\left(t_{j}\right)$ \\
\hline $\mathbf{F}\left(t_{j-1}\right)$ & C & I & & 0 & : & : & $\vdots$ & $\vdots$ & $\mathbf{F}\left(t_{j-1}\right)$ & & $\mathbf{F}\left(t_{j-1}\right)$ \\
\hline . & & C & & & & : & $\vdots$ & $\vdots$ & in & & : \\
\hline $\mathbf{F}\left(t_{1}\right)$ & & $\because$ & & & & & $\vdots$ & $\therefore$ & $\mathbf{F}\left(t_{1}\right)$ & $=\hat{\mathbf{H}}_{*}$ & $\mathbf{F}\left(t_{1}\right)$ \\
\hline $\mathbf{F}\left(t_{0}\right)$ & & $\vdots$ & & .. & $\because$ & I & $\mathbf{O}$ & $\vdots$ & $\mathbf{F}\left(t_{0}\right)$ & & $\mathbf{F}\left(t_{0}\right)$ \\
\hline $\mathbf{Z}\left(t_{0}\right)$ & & $\vdots$ & & & & O & I & $\mathbf{O}$ & $\mathbf{Z}\left(t_{0}\right)$ & & $\mathbf{Z}\left(t_{0}\right)$ \\
\hline $\mathbf{Z}\left(t_{-1}\right)$ & & C & & & O & $a_{3} \mathbf{M}^{-1}$ & $\mathbf{I}-a_{3} \mathbf{M}^{-1} \mathbf{K}$ & $-\left(a_{3} \mathbf{M}^{-1} \mathbf{C}+\Delta t \mathbf{I}\right)$ & $\dot{\mathbf{z}}\left(t_{0}\right)$ & & $\dot{\mathbf{Z}}\left(t_{0}\right)$ \\
\hline
\end{tabular}

By combining Eq. (36) with Eq. (38) we have,

$$
\begin{aligned}
\mathbf{Z}\left(t_{-1}\right) & =a_{3} \mathbf{M}^{-1} \mathbf{F}\left(t_{0}\right)+\left(\mathbf{I}-a_{3} \mathbf{M}^{-1} \mathbf{K}\right) \mathbf{Z}\left(t_{0}\right)-\left(a_{3} \mathbf{M}^{-1} \mathbf{C}+\Delta t \mathbf{I}\right) \dot{\mathbf{Z}}\left(t_{0}\right) \\
& =\left[\begin{array}{lll}
a_{3} \mathbf{M}^{-1} & \mathbf{I}-a_{3} \mathbf{M}^{-1} \mathbf{K} & -\left(a_{3} \mathbf{M}^{-1} \mathbf{C}+\Delta t \mathbf{I}\right)
\end{array}\right]\left[\begin{array}{c}
\mathbf{F}\left(t_{0}\right) \\
\mathbf{Z}\left(t_{0}\right) \\
\dot{\mathbf{Z}}\left(t_{0}\right)
\end{array}\right]
\end{aligned}
$$

6 Subsequently there is,

7 
Denote,

2

$$
\begin{aligned}
\hat{\mathbf{H}} & =\hat{\mathbf{H}}^{*}\left(\prod_{k=1}^{j} \hat{\mathbf{H}}_{k}\right) \hat{\mathbf{H}}_{*} \\
\mathbf{F} & =\left[\begin{array}{llll}
\mathbf{F}^{\mathrm{T}}\left(t_{j}\right) & \ldots & \mathbf{F}^{\mathrm{T}}\left(t_{1}\right) & \mathbf{F}^{\mathrm{T}}\left(t_{0}\right)
\end{array}\right]^{\mathrm{T}}
\end{aligned}
$$

3 Equation (D.1) then can be rewritten in the following form,

4

$$
\mathbf{Z}\left(t_{j+1}\right)=\hat{\mathbf{H}}\left[\begin{array}{c}
\mathbf{F} \\
\mathbf{Z} \\
\dot{\mathbf{Z}}_{0}
\end{array}\right]
$$

5 Through partitioning the matrix $\hat{\mathbf{H}}$ into two parts, namely, $\hat{\mathbf{H}}=\left[\begin{array}{ll}\hat{\mathbf{H}}_{\mathbf{F}} & \hat{\mathbf{H}}_{\mathbf{z}}\end{array}\right]$, the above 6 equation can be rewritten as,

$$
\mathbf{Z}\left(t_{j+1}\right)=\hat{\mathbf{H}}_{\mathbf{F}} \mathbf{F}+\hat{\mathbf{H}}_{\mathbf{Z}}\left[\begin{array}{c}
\mathbf{Z}\left(t_{0}\right) \\
\dot{\mathbf{Z}}\left(t_{0}\right)
\end{array}\right]
$$

8 In the above formulations, the external forces' series in vector $\mathbf{F}$ have the following format,

9

$$
\mathbf{F}=\left[\begin{array}{lllllllllllll}
F_{1}\left(t_{j}\right) & F_{2}\left(t_{j}\right) & \ldots & F_{N}\left(t_{j}\right) & F_{1}\left(t_{j-1}\right) & F_{2}\left(t_{j-1}\right) & \ldots & F_{N}\left(t_{j-1}\right) & \ldots & F_{1}\left(t_{0}\right) & F_{2}\left(t_{0}\right) & \ldots & F_{N}\left(t_{0}\right)
\end{array}\right]^{\mathrm{T}}
$$

While in practice the external forces or excitations are generally represented as $n$ series and each stands for a single excitation, such as,

$$
\begin{aligned}
\mathbf{F}_{1} & =\left[\begin{array}{llll}
F_{1}\left(t_{j}\right) & F_{1}\left(t_{j-1}\right) & \ldots & F_{1}\left(t_{0}\right)
\end{array}\right]^{\mathrm{T}} \\
\mathbf{F}_{2} & =\left[\begin{array}{llll}
F_{2}\left(t_{j}\right) & F_{2}\left(t_{j-1}\right) & \ldots & F_{2}\left(t_{0}\right)
\end{array}\right]^{\mathrm{T}} \\
& \vdots \\
\mathbf{F}_{N} & =\left[\begin{array}{llll}
F_{N}\left(t_{j}\right) & F_{N}\left(t_{j-1}\right) & \ldots & F_{N}\left(t_{0}\right)
\end{array}\right]^{\mathrm{T}}
\end{aligned}
$$

Hence the coefficient matrix $\hat{\mathbf{H}}_{\mathbf{F}}$ should be reordered correspondingly and the displacements' vector $\mathbf{Z}\left(t_{j+1}\right)$ at time point $t_{j+1}$ can be expressed as,

$$
\mathbf{Z}\left(t_{j+1}\right)=\hat{\mathbf{H}}_{\mathbf{F}}^{*} \mathbf{F}^{*}+\hat{\mathbf{H}}_{\mathbf{Z}}\left[\begin{array}{c}
\mathbf{Z}\left(t_{0}\right) \\
\dot{\mathbf{Z}}\left(t_{0}\right)
\end{array}\right]
$$

where $\hat{\mathbf{H}}_{\mathbf{F}}^{*}$ is the matrix after reordering of $\hat{\mathbf{H}}_{\mathbf{F}}$ and $\mathbf{F}^{*}$ is the external force vector ordered in the following form, 
2 Assume that the partitioned form of $\hat{\mathbf{H}}_{\mathbf{F}}^{*}$ is $\hat{\mathbf{H}}_{\mathbf{F}}^{*}=\left[\begin{array}{lllll}\hat{\mathbf{H}}_{\mathbf{F}_{1}}^{*} & \hat{\mathbf{H}}_{\mathbf{F}_{2}}^{*} & \ldots & \hat{\mathbf{H}}_{\mathbf{F}_{N}}^{*}\end{array}\right]$, finally the

3 displacements of the masses at time point $t_{j+1}$ can be represented by the summation of linear

4 combinations of external force time series and the initial conditions of the MDOF system, i.e.,

$$
\mathbf{Z}\left(t_{j+1}\right)=\sum_{k=1}^{N} \hat{\mathbf{H}}_{\mathbf{F}_{k}}^{*} \mathbf{F}_{k}+\hat{\mathbf{H}}_{\mathbf{Z}}\left[\begin{array}{c}
\mathbf{Z}\left(t_{0}\right) \\
\dot{\mathbf{Z}}\left(t_{0}\right)
\end{array}\right]
$$

\section{$6 \quad$ References}

7 [1] O. Ditlevsen, H.O. Madsen, Structural Reliability Methods, John Wiley \& Sons, Chichester, 1996.

[2] R.E. Melchers, Structural Reliability: Analysis and Prediction, second ed. John Wiley \& Sons, Chichester, 1999.

[3] M. Lemaire, Structural Reliability, ISTE-Wiley, New York, 2009.

[4] R.G. Sexsmith, Probability-based safety analysis - value and drawbacks, Structural Safety 21 (1999) 303-310.

[5] Y. Ben-Haim, I. Elishakoff, Convex Models of Uncertainties in Applied Mechanics, Elsevier Science Publisher, Amsterdam, 1990.

[6] Y. Ben-Haim, Convex models of uncertainty in radial pulse buckling of shells, ASME Journal of Applied Mechanics 60 (3) (1993) 683-688.

[7] I. Elishakoff, P. Elisseeff, Non-probabilistic, convex-theoretic modeling of scatter in material properties, AIAA Journal 32 (4) (1994) 843-849.

[8] I. Elishakoff, An idea on the uncertainty triangle, Shock and Vibration Digest 22 (10) (1995) 1. 
1 [9] M. Lombardi, R.T. Haftka, Anti-optimization technique for structural design under load uncertainties, Computer Methods in Applied Mechanics and Engineering 157 (1-2) (1998) $19-31$.

[10] A. Van Wamelen, E.R. Johnson, R.T. Haftka, Optimal design of laminated specimens to evaluate composite failure criteria, Presented at ASC 8th Technical Conference on Composite Materials, Cleveland, 1993.

[11] S. Ganzerli, C.P. Pantelides, Optimum structural design via convex model superposition, Computers \& Structures 74 (2000) 639-647.

[12] L. Fryba, N. Yoshikawa, Bounds analysis of a beam based on the convex model of uncertain foundation, Journal of Sound and Vibration 212(3) (1998) 547-557.

[13] Z.P. Qiu, X.J. Wang, Parameter perturbation method for dynamic responses of structures with uncertain-but-bounded parameters based on interval analysis, International Journal of Solids and Structures 42(18-19) (2005) 4958-4970.

[14] C. Wang, Z.P. Qiu, An interval perturbation method for exterior acoustic field prediction with uncertain-but-bounded parameters, Journal of Fluids and Structures 49 (2014) 441-449.

[15] D. Moens, D. Vandepitte, Recent advances in non-probabilistic approaches for non-deterministic dynamic finite element analysis, Archives of Computational Methods in Engineering 13 (3) (2006) 389-464.

[16] W. Gao, Interval finite element analysis using interval factor method, Computational Mechanics 39 (2007) 709-717.

[17] R.L. Muhanna, H. Zhang, R.L. Mullen, Interval finite element as a basis for generalized 
models of uncertainty in engineering mechanics, Reliable Computing 13 (2) (2007) $173-194$.

[18] D. Moens, M. De Munck, W. Desmet, D. Vandepitte, Numerical dynamic analysis of uncertain mechanical structures based on interval fields, Proceedings of IUTAM Symposium on Vibration Analysis of Structures with Uncertainties, Saint Petersburg, 2009.

[19] W. Verhaeghe, W. Desmet, D. Vandepitte, D. Moens, Interval fields to represent uncertainty on the output side of a static FE analysis, Computer Methods in Applied Mechanics and Engineering 260 (2013) 50-62.

[20] L.P. Zhu, I. Elishakoff, J.H. Starnes Jr. Derivation of multi-dimensional ellipsoidal convex model for experimental data, Mathematical and Computer Modelling 24 (2) (1996) 103-14.

[21] C. Jiang, X. Han, G.Y. Lu, J. Liu, Z. Zhang, Y.C. Bai, Correlation analysis of non-probabilistic convex model and corresponding structural reliability technique, Computer Methods in Applied Mechanics and Engineering 200 (33-36) (2011) $2528-2546$.

[22] Z.P. Qiu, X.J. Wang, Two non-probabilistic set-theoretical models for dynamic response and buckling failure measures of bars with unknown-but-bounded initial imperfections, International Journal of Solids and Structures 42 (3-4) (2005) 1039-1054.

[23] I. Elishakoff, Y. Bekel, Application of Lamé's super ellipsoids to model initial imperfections, ASME Journal of Applied Mechanics 80 (2013) 061006-1.

[24] Y.J. Luo, Z. Kang, Z. Luo, A. Li, Continuum topology optimization with 
non-probabilistic reliability constraints based on multi-ellipsoid convex model, Structural and Multidisciplinary Optimization 39 (3) (2009) 297-310.

[25] Z. Kang, Y.J. Luo, Non-probabilistic reliability-based topology optimization of geometrically nonlinear structures using convex models, Computer Methods in Applied Mechanics and Engineering 198 (41-44) (2009) 3228-3238.

[26] C. Jiang, Q.F. Zhang, X. Han, Multidimensional parallelepiped model - a new type of non-probabilistic convex model for structural uncertainty quantification, International Journal for Numerical Methods in Engineering 103 (1) (2015) 31-59.

[27] Y. Ben-Haim, A non-probabilistic measure of reliability of linear systems based on expansion of convex models, Structural Safety 17 (1995) 91-109.

[28] H.J. Cao, B.Y. Duan, An approach on the non-probabilistic reliability of structures based on uncertainty convex models, Chinese Journal of Computational Mechanics 22 (5) (2005) 546-549.

[29] C. Jiang, R.G. Bi, G.Y. Lu, X. Han, Structural reliability analysis using non-probabilistic convex model, Computer Methods in Applied Mechanics and Engineering 254 (2013) 83-98.

[30] C. Jiang, Q.F. Zhang, X. Han, Y.H. Qian, A non-probabilistic structural reliability analysis method based on a multidimensional parallelepiped convex model, Acta Mechanica 225 (2) (2014) 383-395.

[31] X.J. Wang, Y. Xia, X.Q. Zhou, Y. Chen, Structural damage measure index based on non-probabilistic reliability model, Journal of Sound and Vibration 333 (5) (2014) 1344-1355. 
[32] X.P. Du, Interval reliability analysis, Proceedings of the ASME 2007 International Design Engineering Technical Conferences \& Computers and Information in Engineering Conference, Las Vegas, Nevada, USA, 2007, pp. 1103-1109.

[33] W. Gao, C. M. Song, F. Tin-Loi, Probabilistic interval analysis for structures with uncertainty, Structural Safety 32 (3) (2010) 191-199.

[34] X.F. Yang, Y.S. Liu, Y.S. Zhang, Z.F. Yue, Probability and convex set hybrid reliability analysis based on active learning Kriging model, Applied Mathematical Modelling 39 (14) (2015) 3954-3971.

[35] X. Guo, W. Bai, W.S. Zhang, X.X. Gao, Confidence Structural Robust Design and Optimization Under Stiffness and Load Uncertainties, Computer Methods in Applied Mechanics and Engineering 198 (41) (2009) 3378-3399.

[36] C. Jiang, Z.G. Zhang, Q.F. Zhang, X. Han, H.C. Xie, J. Liu, A new nonlinear interval programming method for uncertain problems with dependent interval variables, European Journal of Operational Research 238 (1) (2014) 245-253.

[37] S.M. Ross, Stochastic processes, John Wiley \& Sons, New York, 1983.

[38] Sidney I. Resnick, Adventures in stochastic processes, Birkhäuser, Boston, 1992.

[39] C. Jiang, B.Y. Ni, X. Han, Y.R. Tao, Non-probabilistic convex model process: A new method of time-variant uncertainty analysis and its application to structural dynamic reliability problems, Computer Methods in Applied Mechanics and Engineering 268 (2014) 656-676.

[40] S. Ferson, V. Kreinovich, J. Hajagos, W. Oberkampf, L. Ginzburg, Experimental uncertainty estimation and statistics for data having interval uncertainty, Sandia National 
[41] X.P. Du, A. Sudjianto, B.Q. Huang, Reliability-based design with the mixture of random and interval variables, ASME Journal of Mechanical Design 127 (2005) 1068-1076.

[42] R.E. Moore, R.B. Kearfott, M.J. Cloud, Introduction to interval analysis, Society for Industrial and Applied Mathematics, Philadelphia, 2009.

[43] S.S. Dragomir, A survey on Cauchy-Bunyakovsky-Schwarz type discrete inequalities, Journal of Inequalities in Pure and Applied Mathematics 4 (3) (2003) 1-142.

[45] M.D. Moura, E. Zio, I.D. Lins, E. Droguett, Failure and reliability prediction by support vector machines regression of time series data, Reliability Engineering \& System Safety 99 (11) (2011) 1527-1534.

[46] A.H. Roger, R.J. Charles, Matrix analysis, Cambridge University Press, Cambridge, 1990.

[47] S.H. Crandall, Random vibration, The MIT Press, Cambridge, 1958.

[48] N.C. Nigam, Introduction to random vibrations, The MIT Press, Cambridge, 1983.

[49] W.Q. Zhu, Random vibration, Science Press, Beijing, 1992. (in Chinese)

[50] D. Newland, An introduction to random vibrations, Spectral and Wavelet Analysis, third ed. Longman, New York, 1993.

[51] J. Li, J.B. Chen, Stochastic Dynamics of Structures, John Wiley \& Sons, Asia, 2009.

[52] J.H. Lin, Y.H. Zhang, Y. Zhao, Volume 5 Seismic Design, Chapter 5 "Seismic Random Response Analysis" in Bridge Engineering Handbook, CRC Press, Taylor \& Francis, 
Boca Raton, FL, 2012.

2 [53] S. Timoshenko, D.H. Young, W. Weaver. Jr., Vibration problems in engineering, fourth ed. John Wiley \& Sons, New York, 1974.

4 [54] B. Hamann, J.L. Chen, Data point selection for piecewise linear curve approximation, Computer Aided Geometric Design 11 (3) (1994) 289-301.

[55] R.W. Clough, J. Penzien, Dynamics of structures, Mc-Graw Hill, New York, 1975.

7 [56] S.S. Rao, Mechanical vibrations, Prentice Hall, Englewood Cliffs, 2004.

8 [57] K.J. Bathe, E.L. Wilson, Numerical methods in finite element analysis, Prentice Hall, $9 \quad$ New Jersey, 1976.

[58] M.H. Shojaeefard, A. Khalkhali, P. Safarpour Erfani, Multi-Objective Suspension Optimization of a 5-DOF Vehicle Vibration Model Excited by Random Road Profile, $1-7$. 


\section{$1 \quad$ Figure captions}

$2 \quad$ Fig. 1. Interval process model.

Fig. 2. Three cases of the interval process. (a) With constant middle point function, (b) with

4 constant half width function, (c) with constant variation coefficient function.

5 Fig. 3. Classification of the interval process. (a) Discrete interval process, (b) continuous interval process.

7 Fig. 4. Calculation of the self-correlation function.

8 Fig. 5. Several groups of samples and corresponding values of the self-correlation function. (a) $r_{X X}\left(t_{i}, t_{j}\right)=1$ (b) $r_{X X}\left(t_{i}, t_{j}\right)=-1$,

(c) $r_{X X}\left(t_{i}, t_{j}\right)=0.60$

(d) $r_{X X}\left(t_{i}, t_{j}\right)=-0.67$ $r_{X X}\left(t_{i}, t_{j}\right)=0$

Fig. 6. A stationary interval process.

Fig. 7. SDOF spring-mass-damper vibration system.

Fig. 8. Approximating the excitation with piecewise linear function.

Fig. 9. MDOF spring-mass-damper system.

Fig. 10. Displacement response bounds of the damped SDOF system in different correlation cases. (a) $r(\tau)=\mathrm{e}^{-\alpha|\tau|}, \alpha=0.2$ (b) $r(\tau)=\mathrm{e}^{-\alpha|\tau|} \cos \omega \tau$, (c) $r(\tau)=1-|\tau| / T_{0}, T_{0}=60 \mathrm{~s}$, (d) $r(\tau)=0, \tau \neq 0$, (e) Comparison of four different correlation cases.

Fig. 11. Velocity response bounds of the damped SDOF system in different correlation cases.

Fig. 12. Displacement response bounds of the undamped SDOF system in different correlation cases.

Fig. 13. The excitation force $F^{I}(t)$.

Fig. 14. Displacement response bounds of the damped system when $F^{I}(t)$ is non-stationary. 
(a) $\omega_{F}=4$, (b) $\omega_{F}=1$, (c) $\omega_{F}=0.5$.

Fig. 15. A 3-DOF system.

3 Fig. 16. Displacement response bounds of the damped 3-DOF damped system.

$4 \quad$ Fig. 17. Displacement response bounds of the undamped 3-DOF damped system.

$5 \quad$ Fig. 18. Half vehicle vibration model with 5-DOF [58].

6 Fig. 19. Response bounds of the half vehicle model without consideration of 7 mutual-correlation of road excitations.

8 Fig. 20. Response bounds of the half vehicle model with consideration of mutual-correlation 9 of road excitations.

Fig. 21. Responses of the half vehicle model under a deterministic road excitation.

\section{Figure captions}

Table 1 Several typical self-correlation functions and corresponding sample curves for stationary interval process model

Table 2 Constant parameters of the half vehicle model.

Table 3 Results of vehicle vibration analysis without consideration of mutual-correlation of road excitations.

Table 4 Results of vehicle vibration analysis with consideration of mutual-correlation of road excitations. 


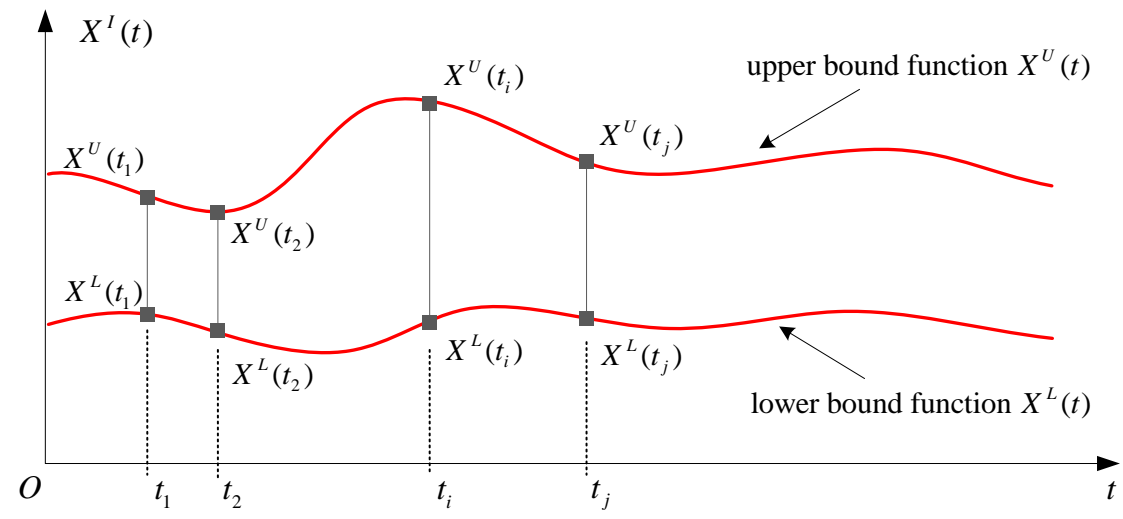

5

Fig. 1. Interval process model.

6

7 
(a)

2

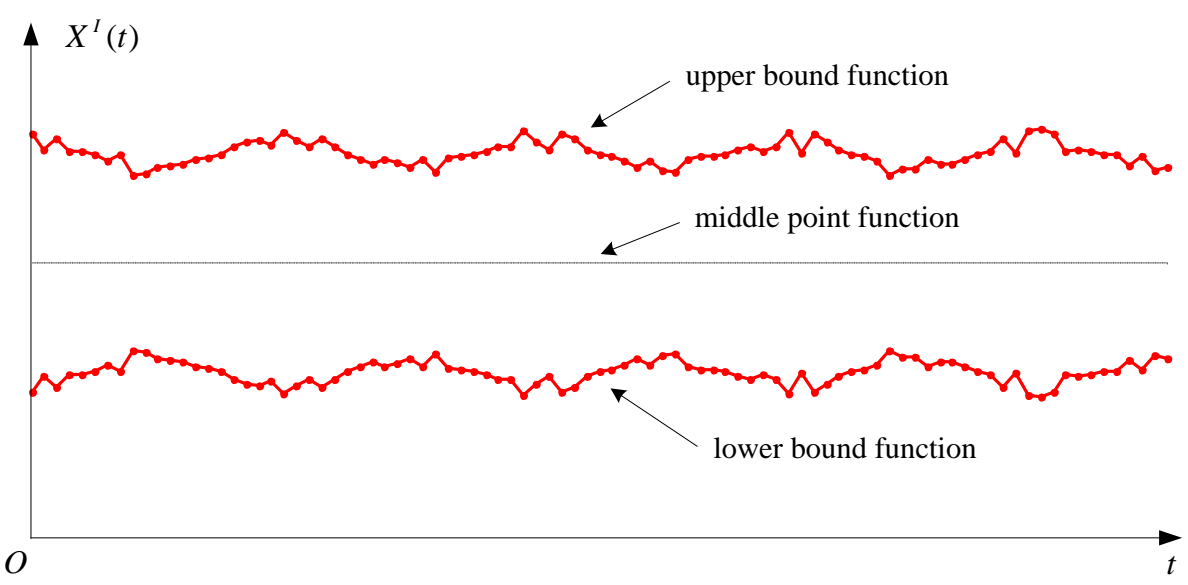

3 (b)

4

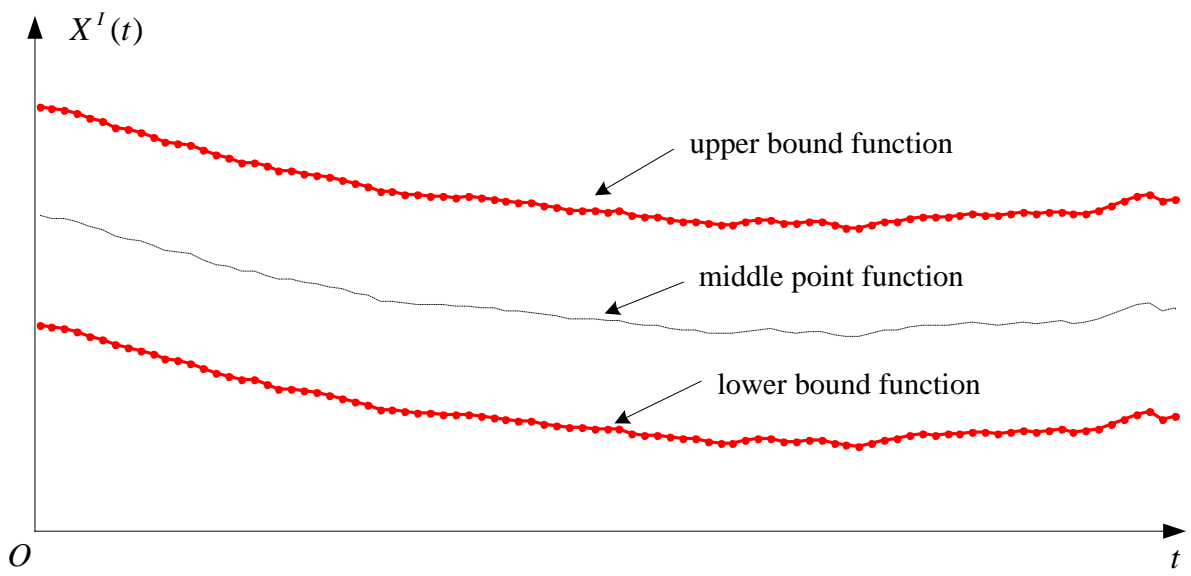

$5 \quad(c)$

6

7

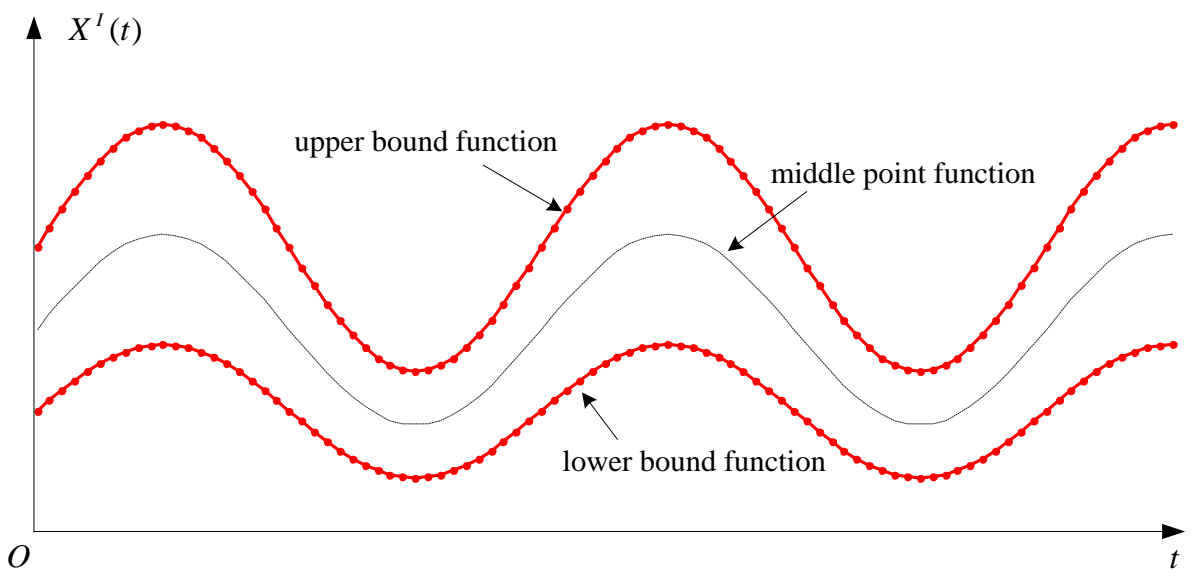

Fig. 2. Three cases of the interval process. (a) With constant middle point function, (b) with constant half width function, (c) with constant variation coefficient function. 


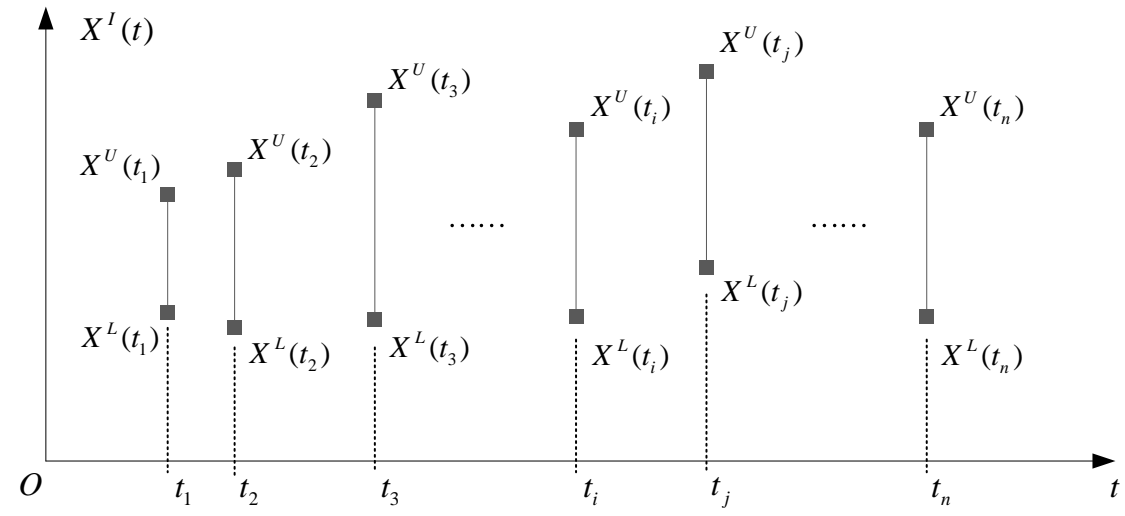

$6 \quad(b)$

7

8

9

10

11

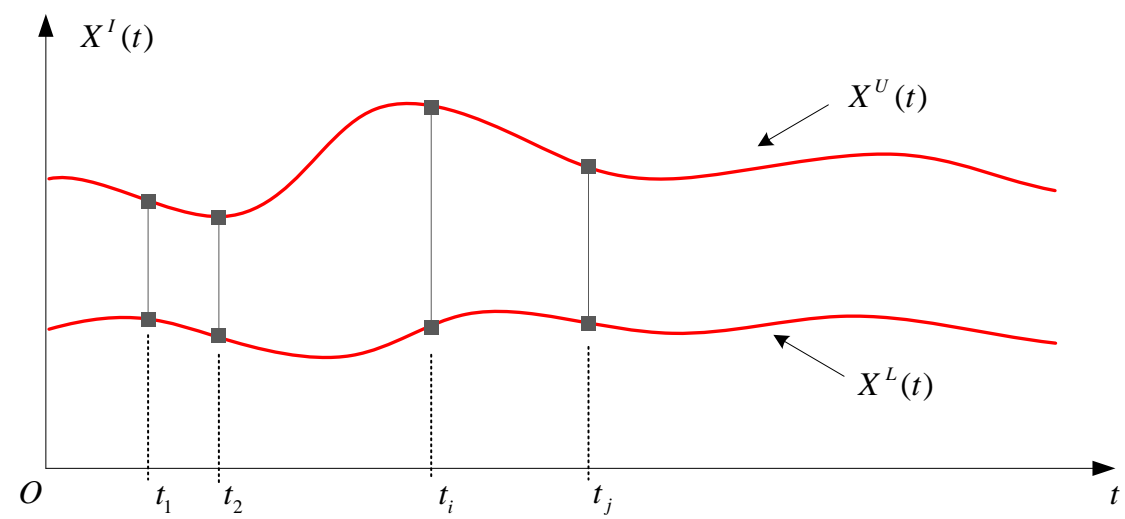

Fig. 3. Classification of the interval process. (a) Discrete interval process, (b) continuous interval process. 


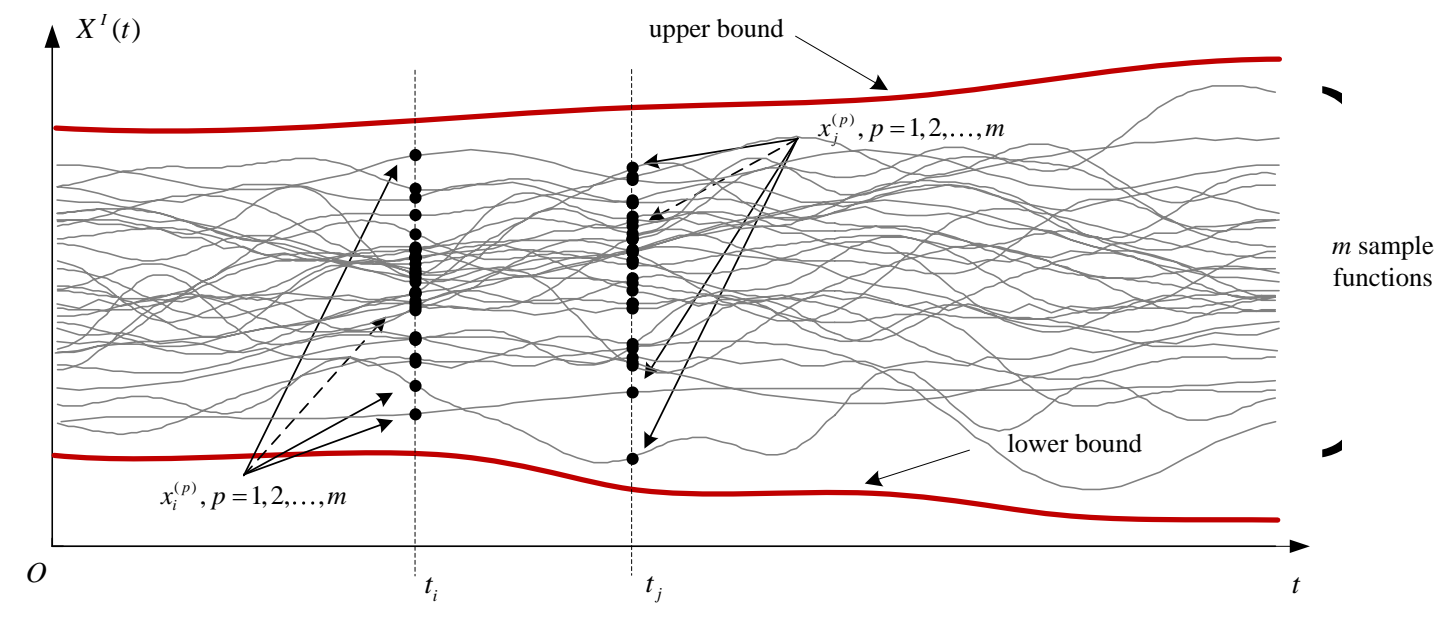

Fig. 4. Calculation of the self-correlation function

6

7 
$4 \quad$ (a)

5

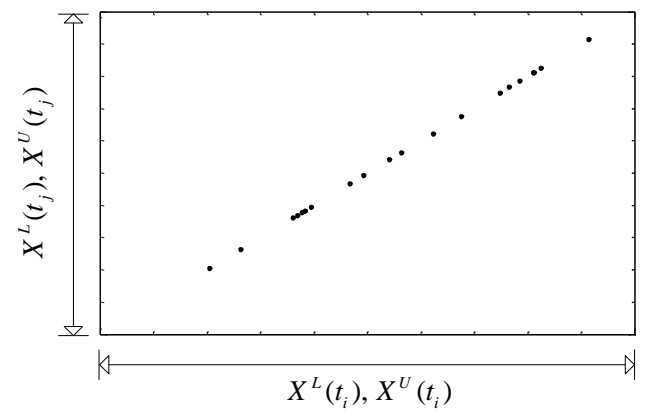

6 (c)

7

8

9

(e) (b)

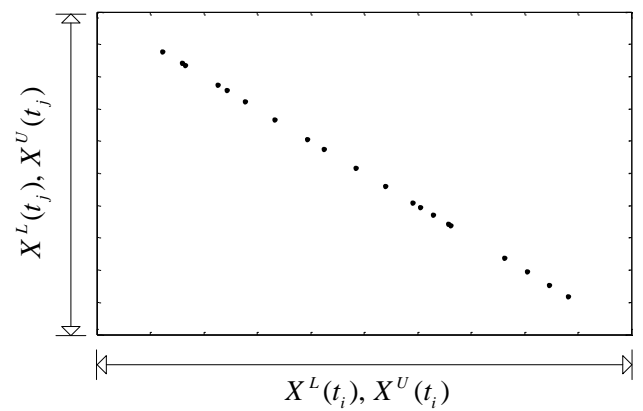

(d)

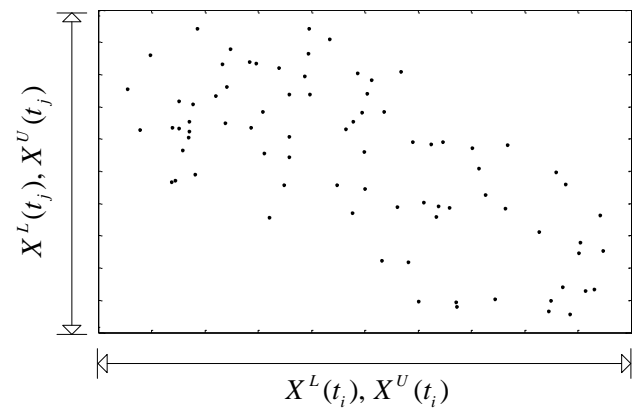

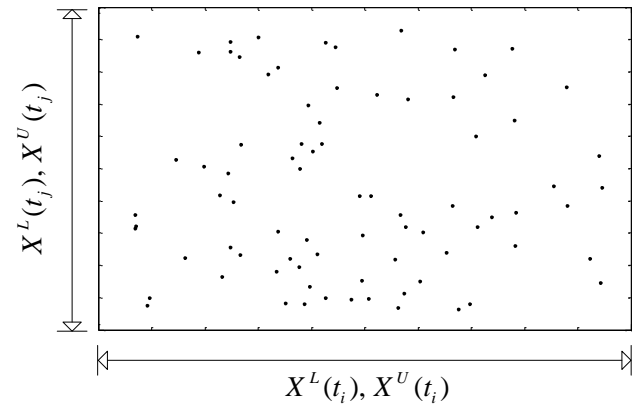

Fig. 5. Several groups of samples and corresponding values of the self-correlation function. (a)

$$
r_{X X}\left(t_{i}, t_{j}\right)=1 \text {, (b) } r_{X X}\left(t_{i}, t_{j}\right)=-1 \text {, (c) } r_{X X}\left(t_{i}, t_{j}\right)=0.60 \text {, (d) } r_{X X}\left(t_{i}, t_{j}\right)=-0.67 \text {, (e) }
$$

$$
r_{X X}\left(t_{i}, t_{j}\right)=0 \text {. }
$$


3

6

7

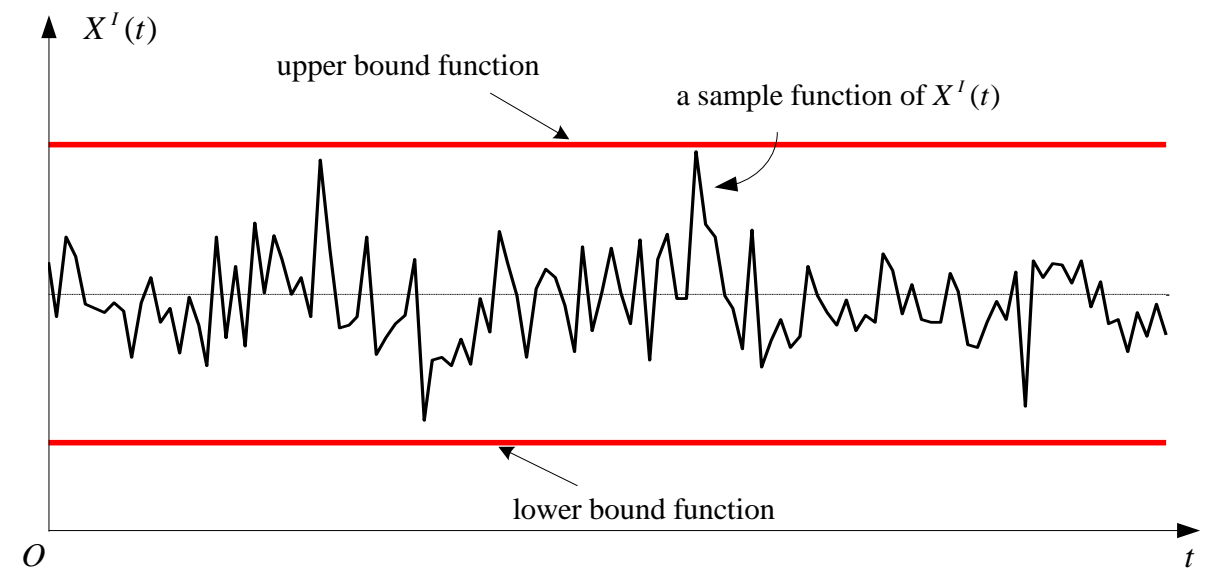

Fig. 6. A stationary interval process 
3

4

(a)

(b)
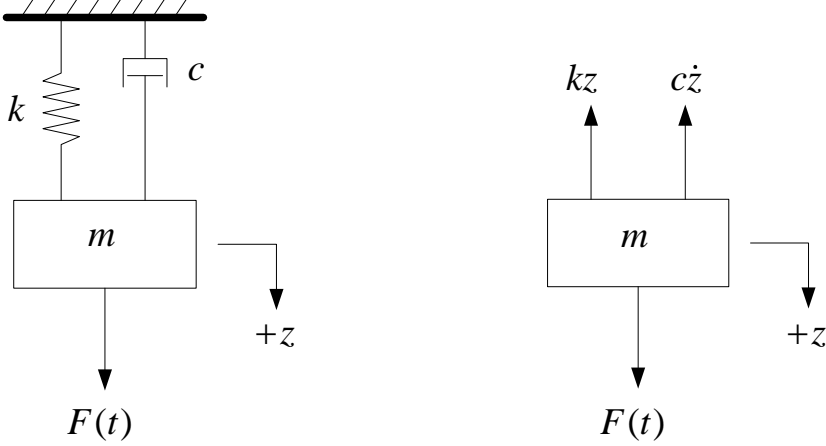

Fig. 7. SDOF spring-mass-damper vibration system

7

8 


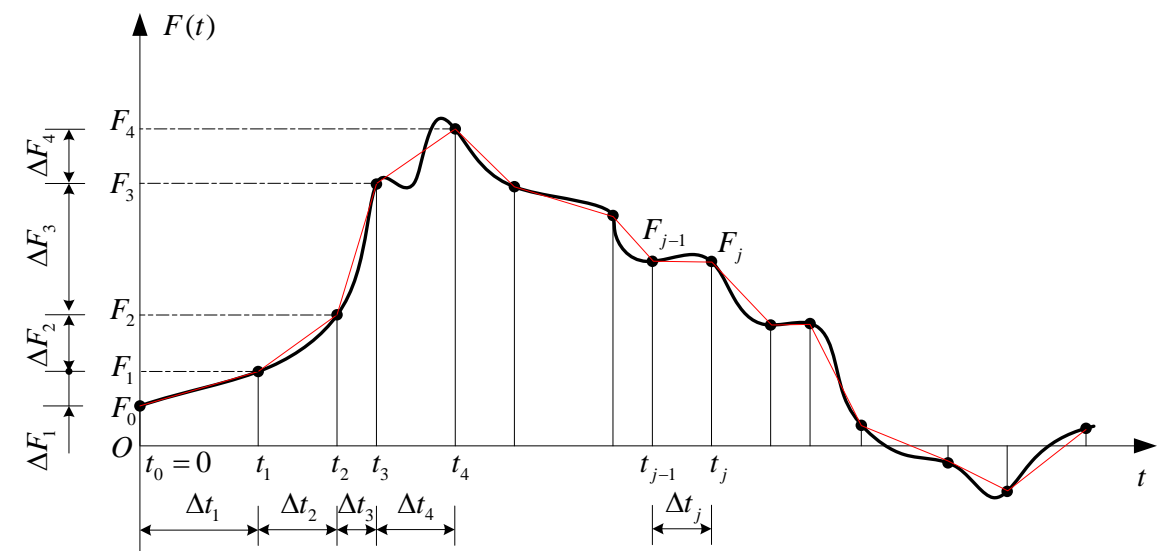

Fig. 8. Approximating the excitation with piecewise linear function

6

7 
$4 \quad$ (a)

5

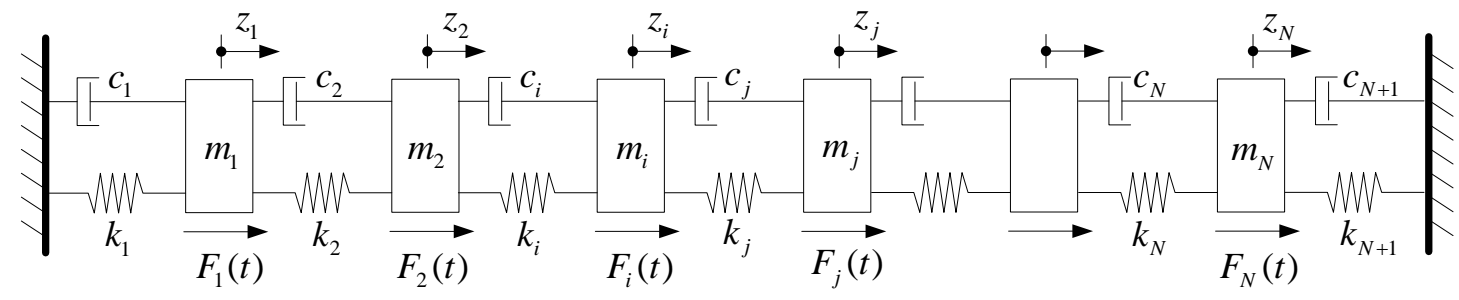

$6 \quad(b)$

7

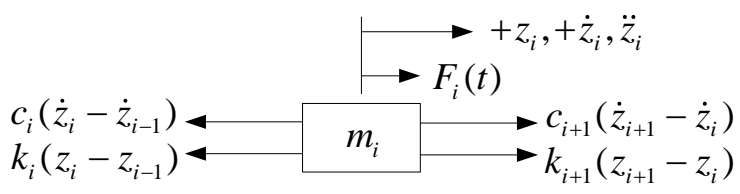

8

Fig. 9. MDOF spring-mass-damper system

9

10 
(a)

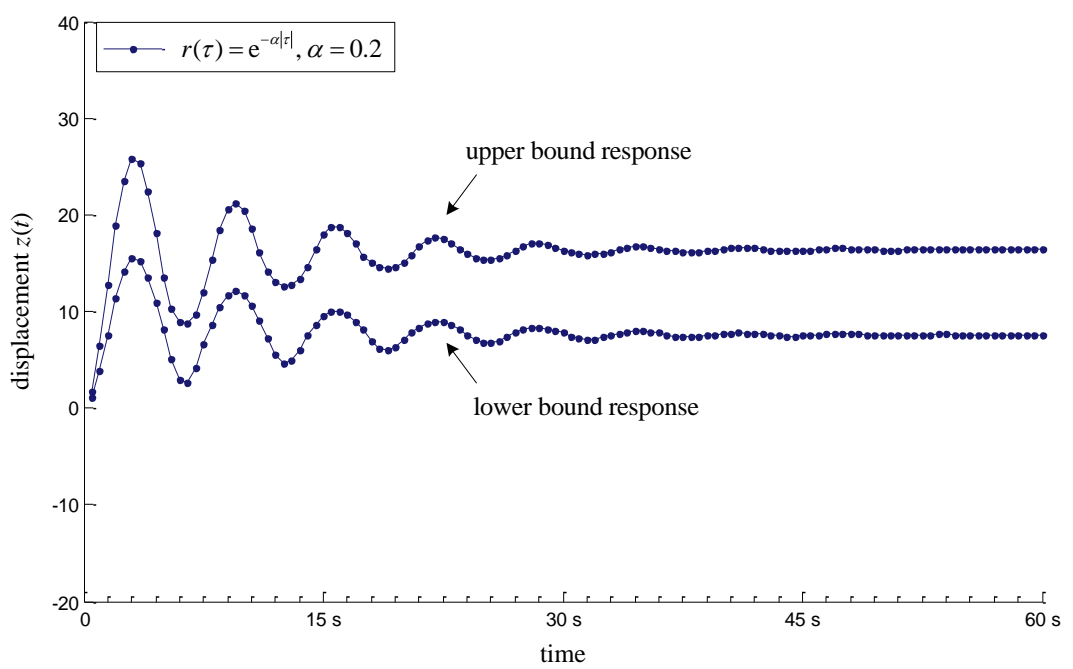

(b)

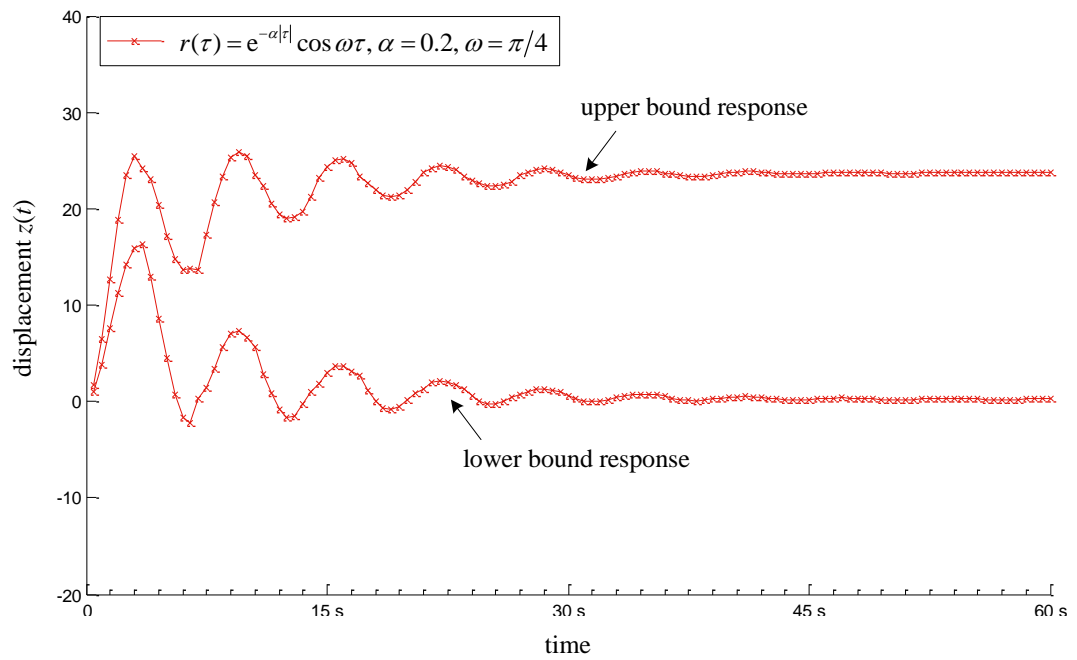

5 (c)

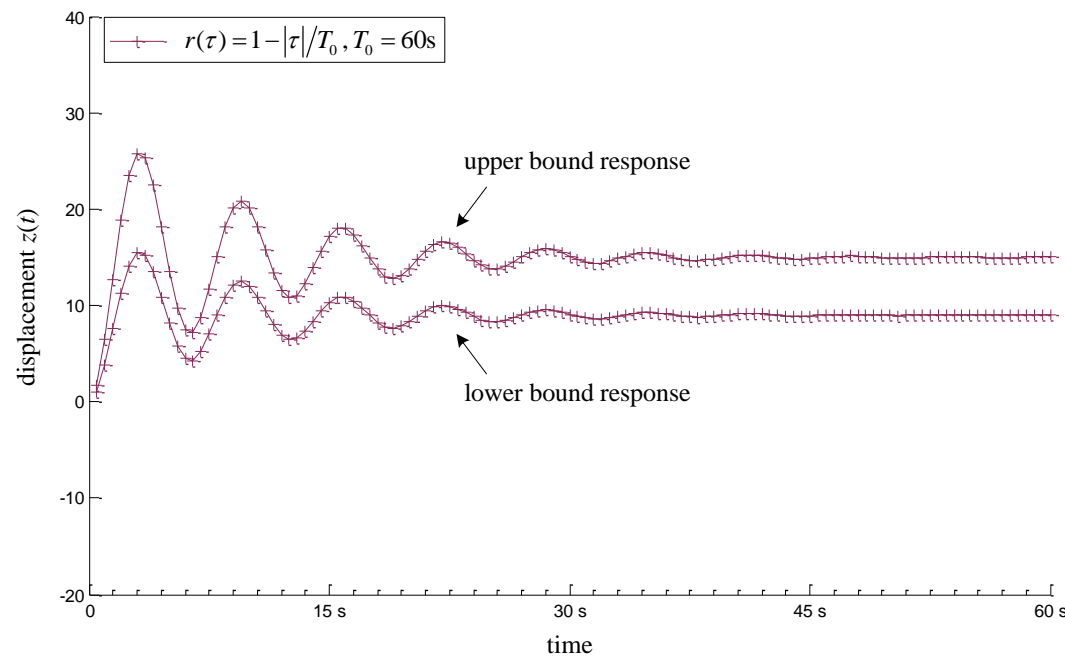




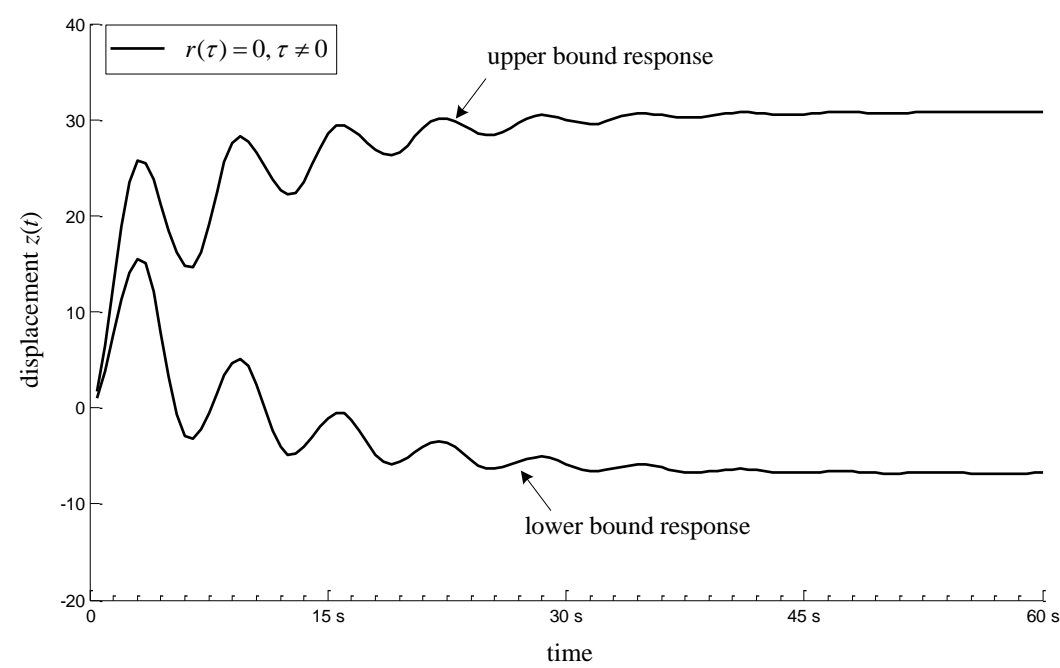

$3(\mathrm{e})$

4

5

6

7

8

9

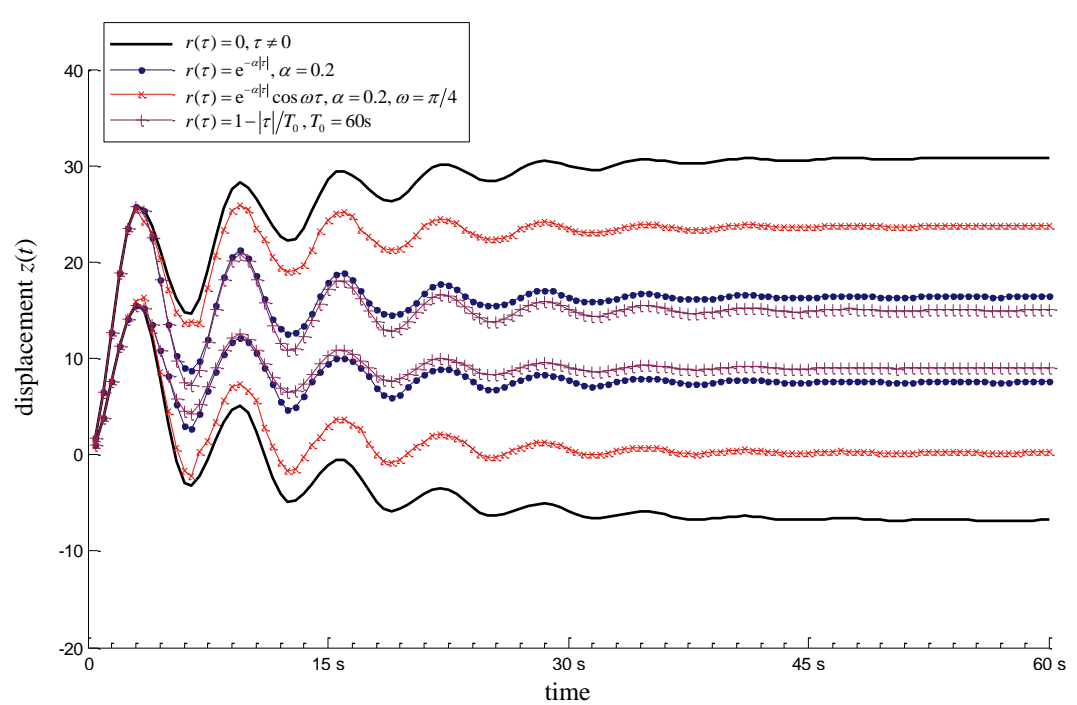

Fig. 10. Displacement response bounds of the damped SDOF system in different correlation cases. (a) $r(\tau)=\mathrm{e}^{-\alpha|\tau|}, \alpha=0.2$, (b) $\quad r(\tau)=\mathrm{e}^{-\alpha|\tau|} \cos \omega \tau$, (c) $\quad r(\tau)=1-|\tau| / T_{0}, T_{0}=60 \mathrm{~s}$, (d) $r(\tau)=0, \tau \neq 0$, (e) Comparison of four different correlation cases. 


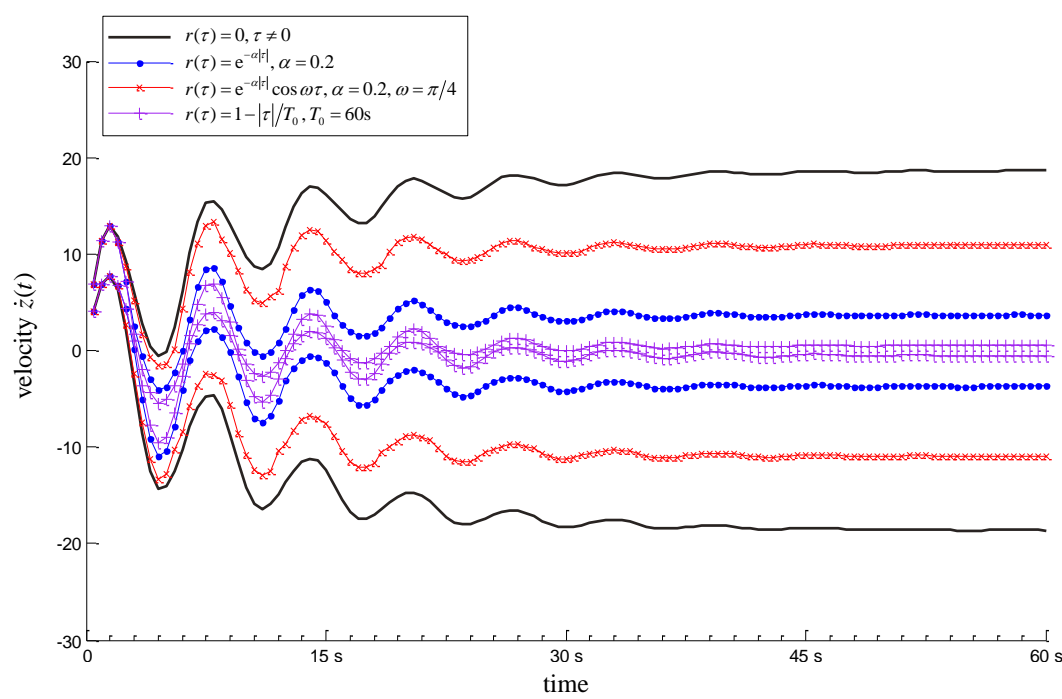

$5 \quad$ Fig. 11. Velocity response bounds of the damped SDOF system in different correlation cases 6 7 


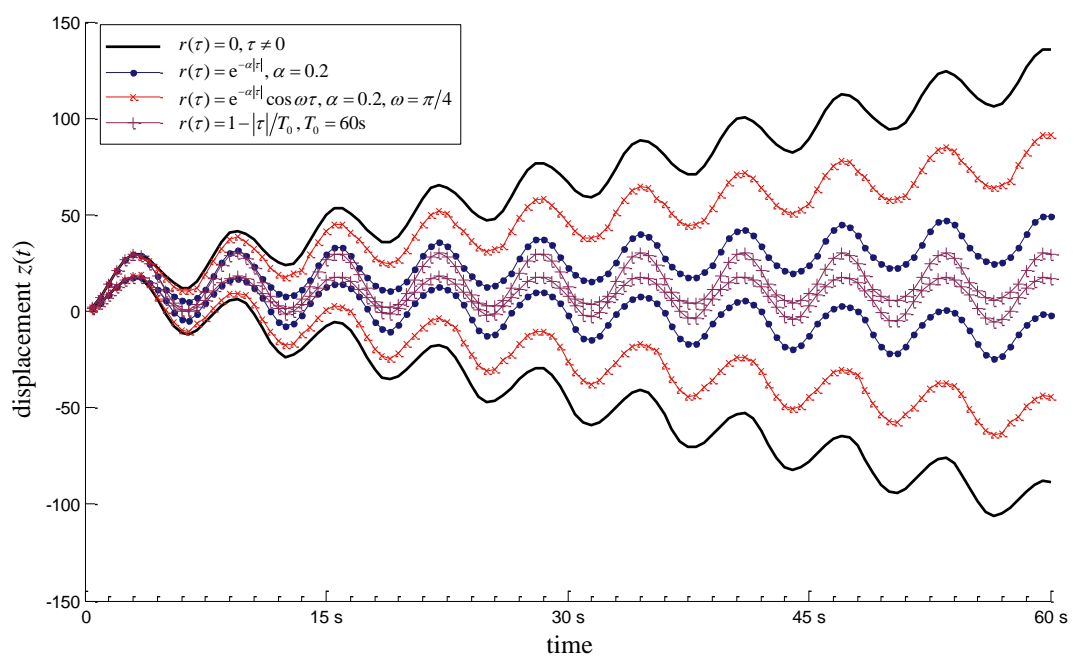

Fig. 12. Displacement response bounds of the undamped SDOF system in different 


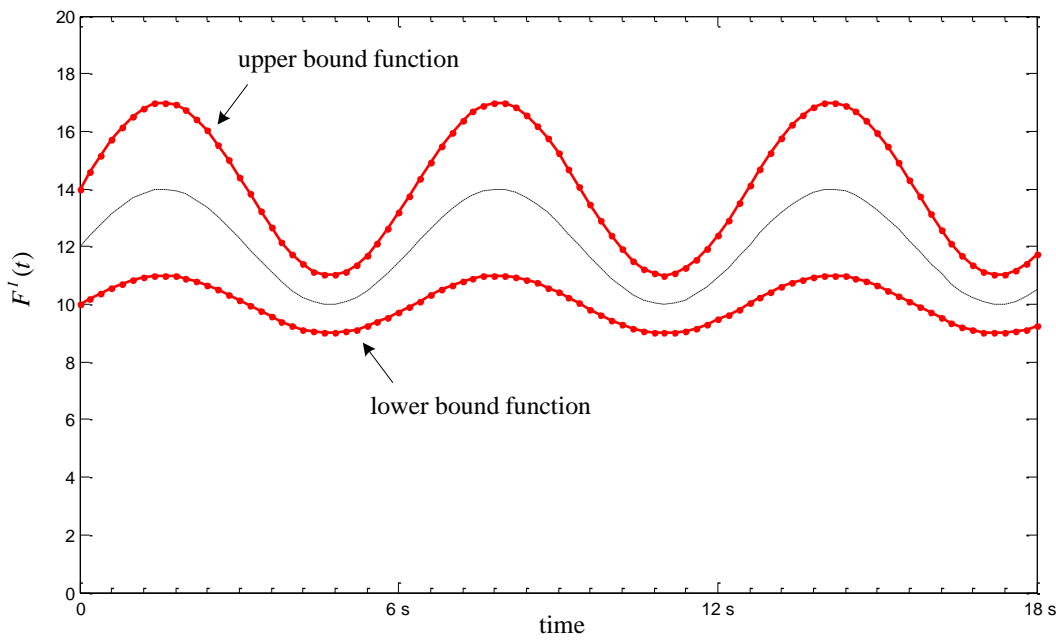

Fig. 13. The excitation force $F^{I}(t)$

6

7 
(a)

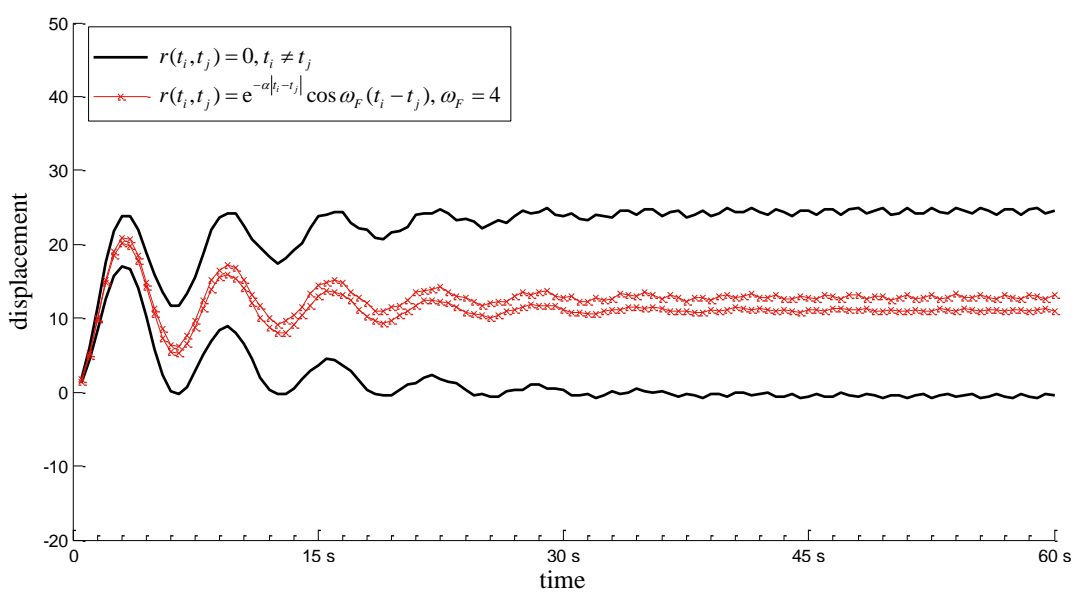

3 (b)

4

5

(c)

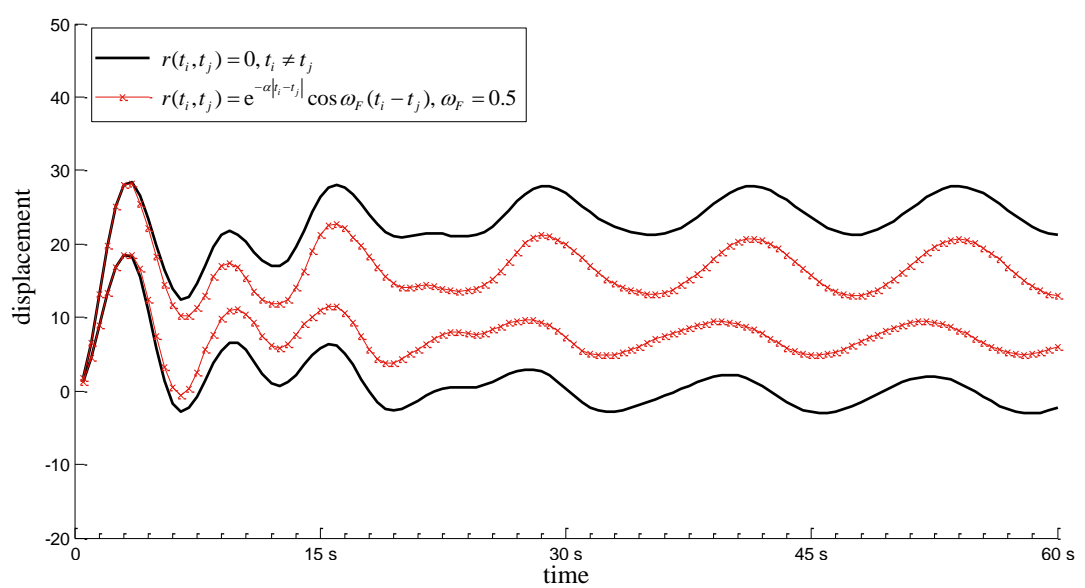

6

7

8

(a) $\omega_{F}=4$, (b) $\omega_{F}=1$, (c) $\omega_{F}=0.5$.

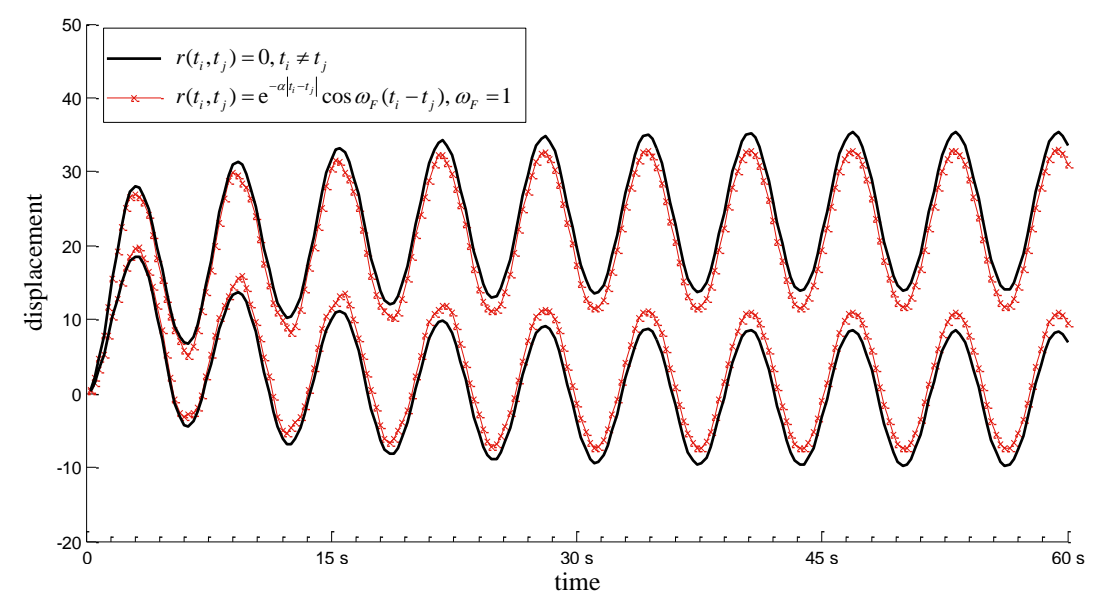

Fig. 14. Displacement response bounds of the damped system when $F^{I}(t)$ is non-stationary. 
3

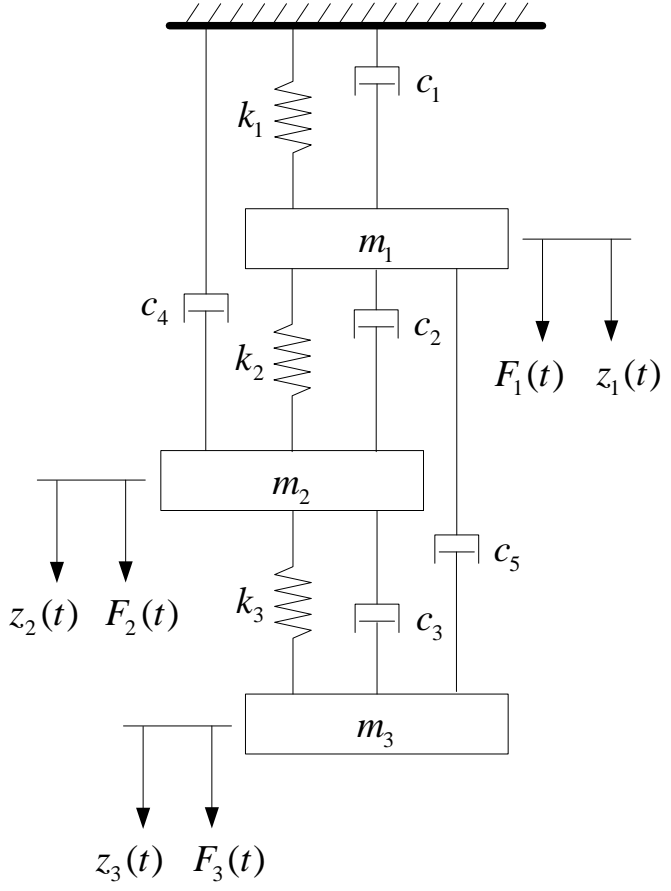

5

Fig. 15. A 3-DOF system

6

7 

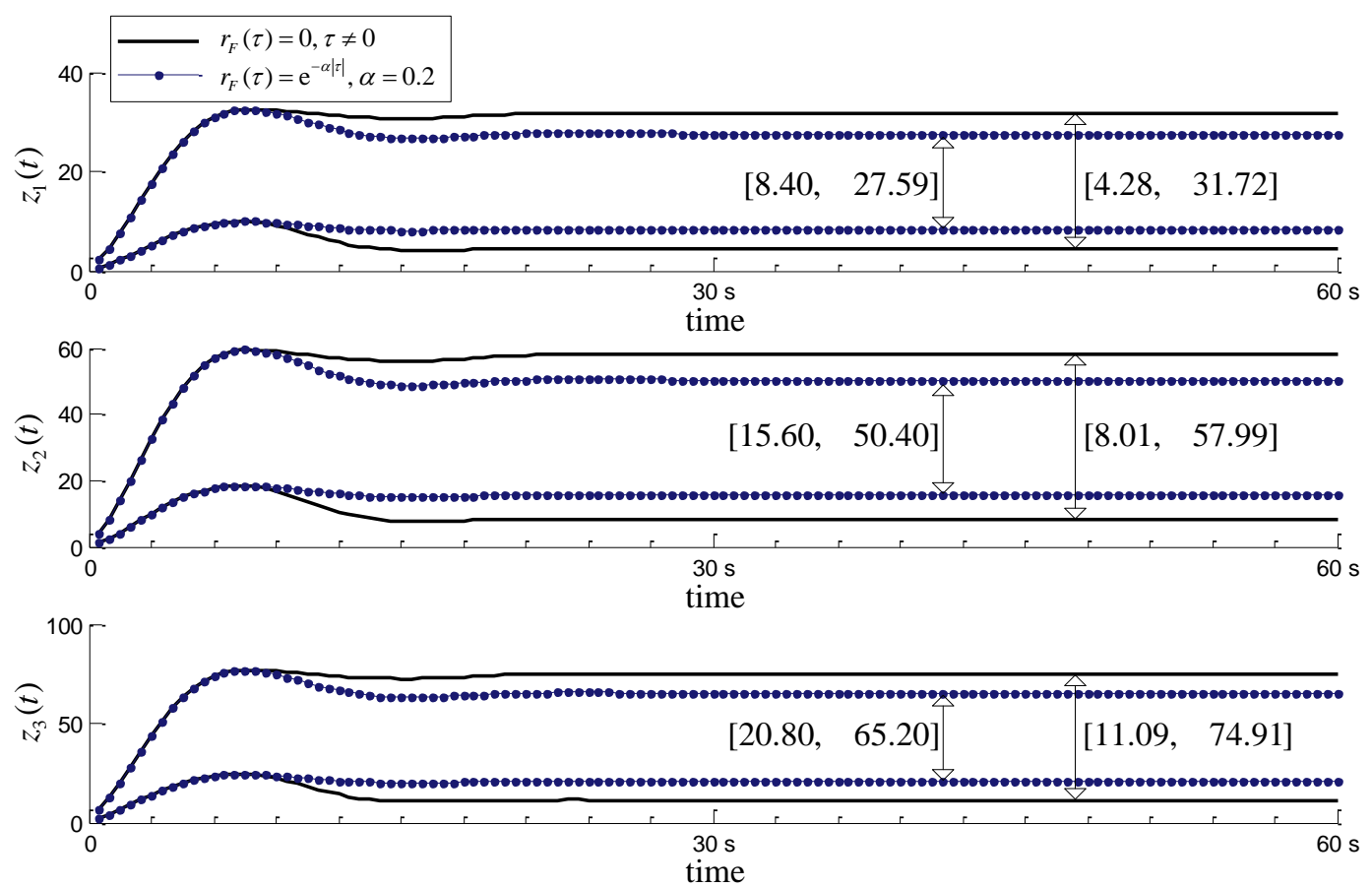

4

Fig. 16. Displacement response bounds of the damped 3-DOF damped system 

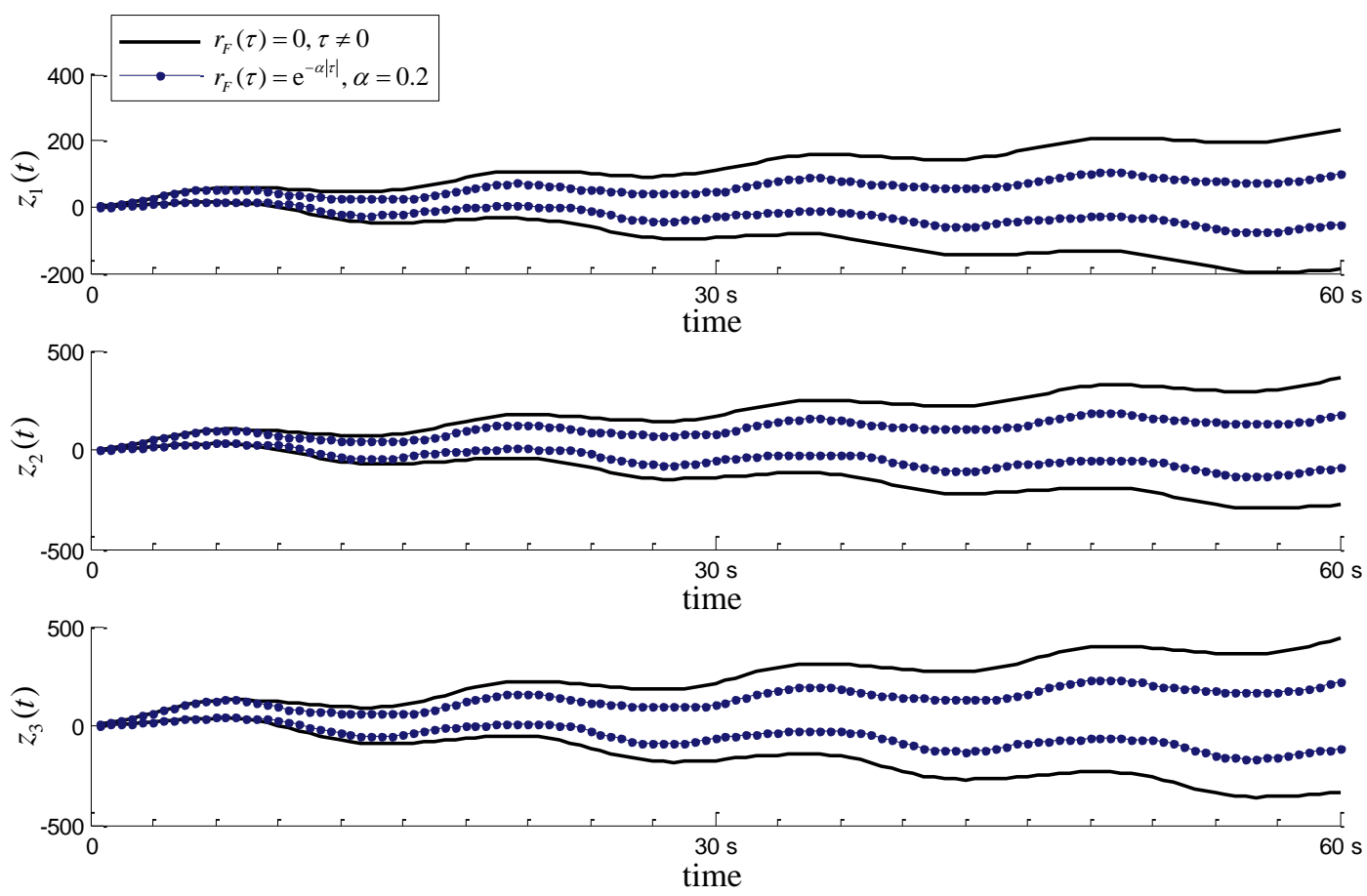

Fig. 17. Displacement response bounds of the undamped 3-DOF damped system 


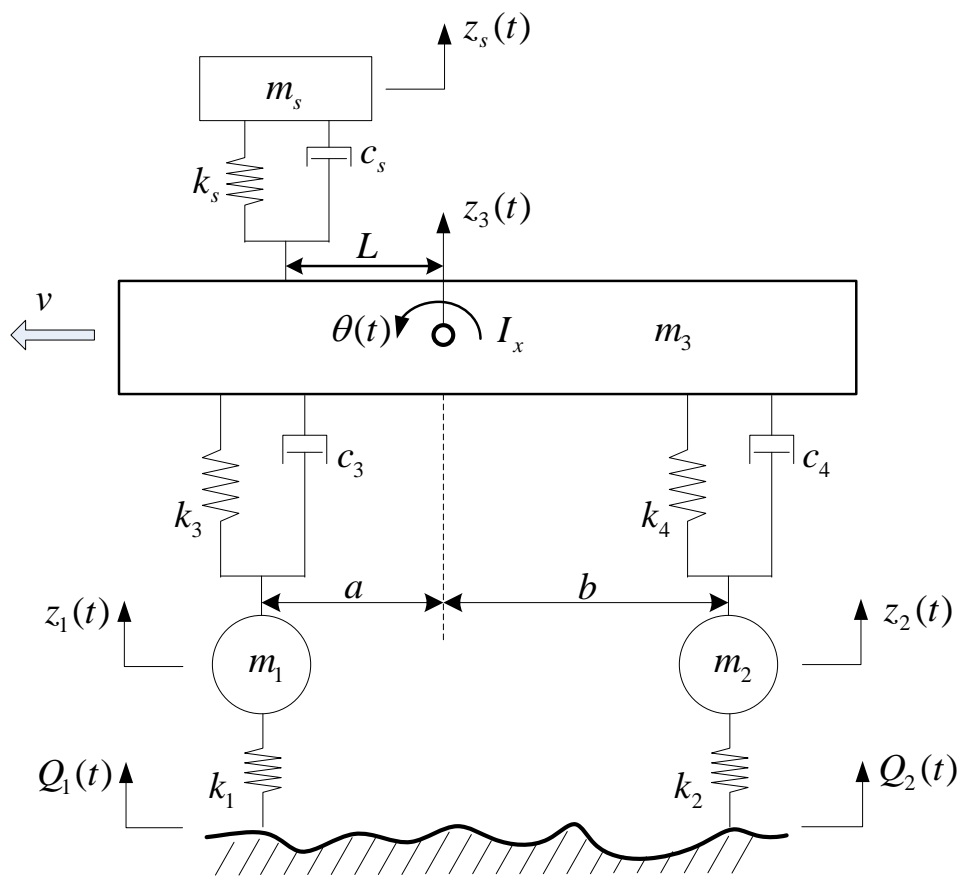

Fig. 18. Half vehicle vibration model with 5-DOF [58]

6

7 

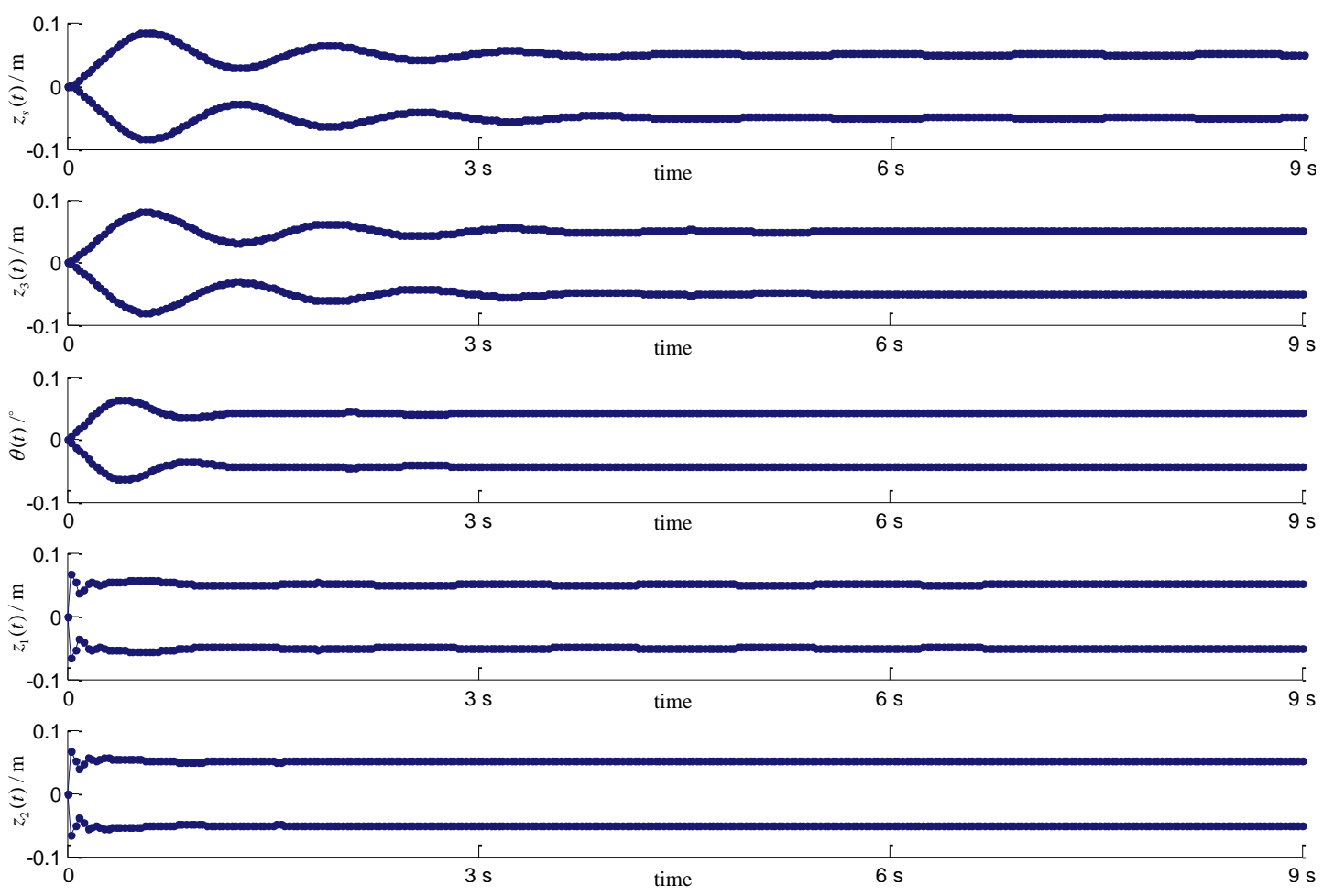

4

5

Fig. 19. Response bounds of the half vehicle model without consideration of

mutual-correlation of the road excitations

7

8 

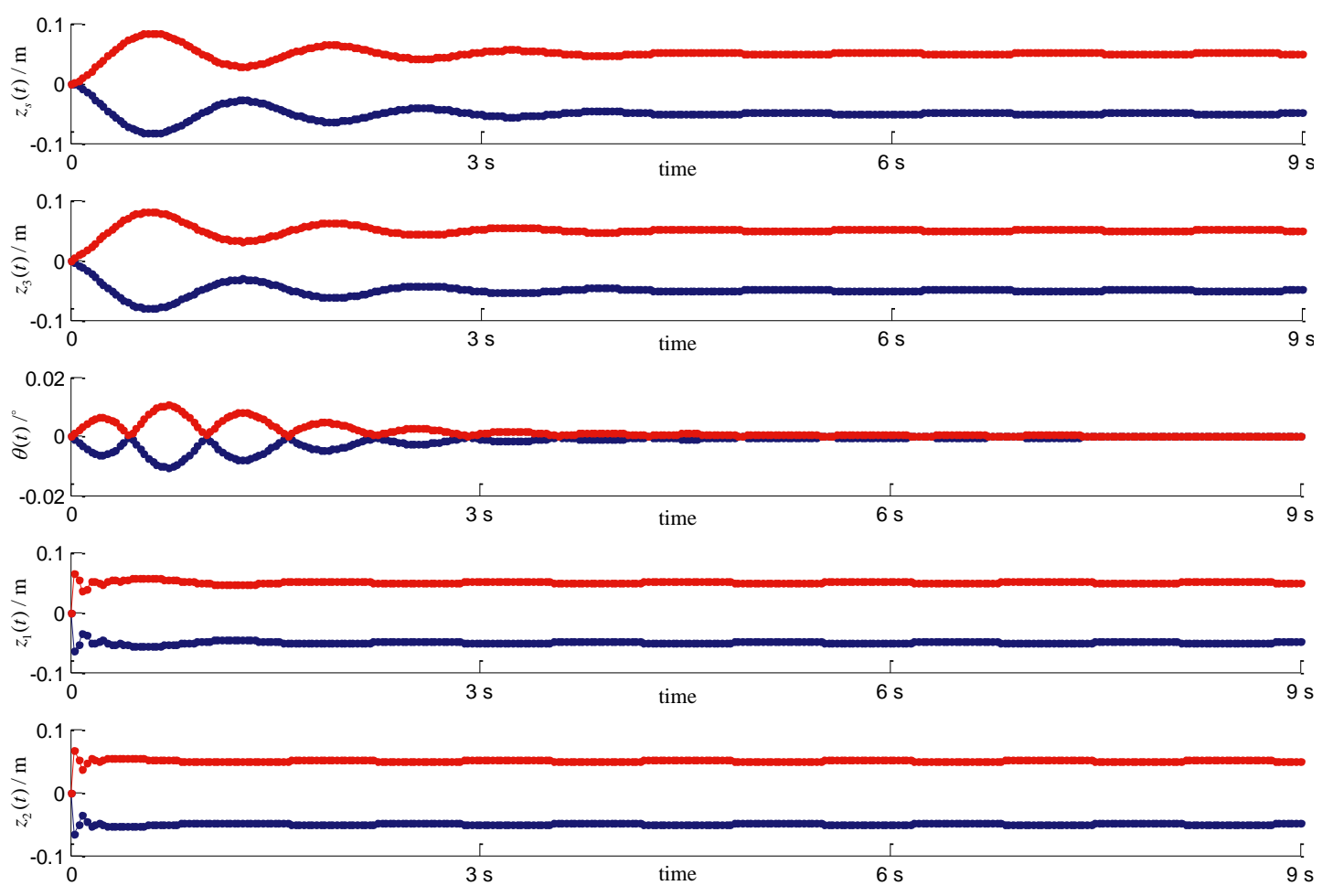

4

5 Fig. 20. Response bounds of the half vehicle model with consideration of mutual-correlation

of the road excitations

7

8 
3

4

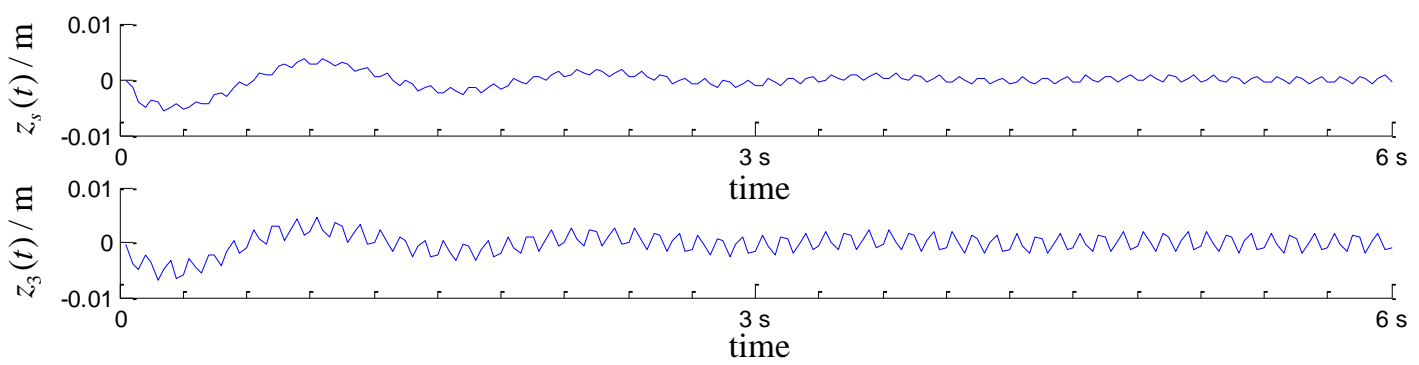

5

Fig. 21. Responses of the half vehicle model under a deterministic road excitation

6

7 
2 Several typical self-correlation functions and corresponding sample curves for stationary

3 interval process model

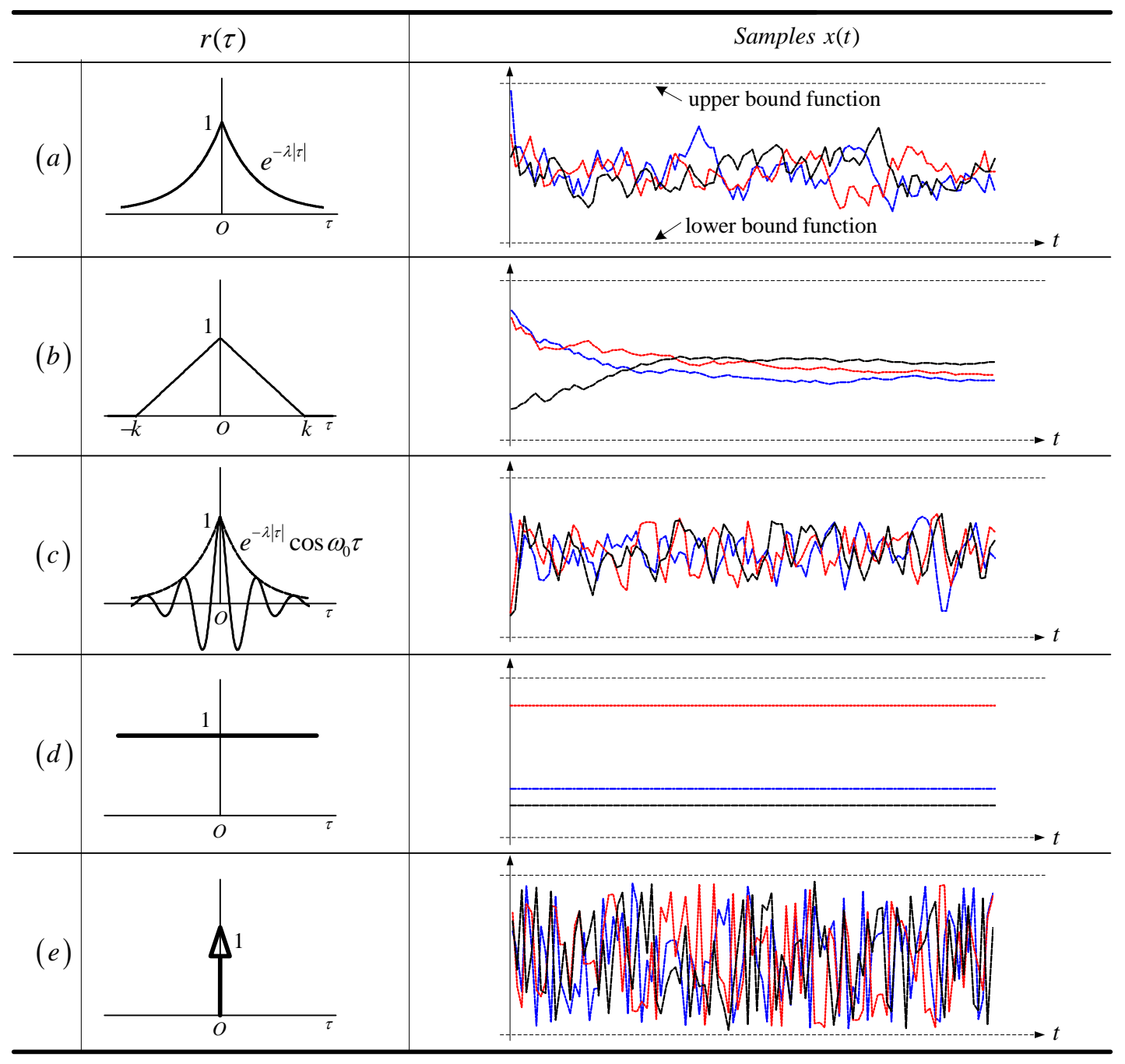

5 
Table 2

3 Constant parameters of the half vehicle model

\begin{tabular}{|c|c|c|}
\hline$m_{s}$ & mass of driver and chair & $75 \mathrm{~kg}$ \\
\hline$m_{1}$ & mass of front wheel & $59 \mathrm{~kg}$ \\
\hline$m_{2}$ & mass of rack wheel & $53 \mathrm{~kg}$ \\
\hline$m_{3}$ & sprung mass & $1000 \mathrm{~kg}$ \\
\hline$I_{x}$ & moment of inertia of the vehicle body around the horizontal axis & $803 \mathrm{~kg} \cdot \mathrm{m}^{2}$ \\
\hline$c_{s}$ & damping coefficient of chair & $1578 \mathrm{~N} /(\mathrm{m} / \mathrm{s})$ \\
\hline$c_{3}$ & damping coefficient of front suspension & $1140 \mathrm{~N} /(\mathrm{m} / \mathrm{s})$ \\
\hline$c_{4}$ & damping coefficient of rear suspension & $1140 \mathrm{~N} /(\mathrm{m} / \mathrm{s})$ \\
\hline$L$ & distance between chair and vehicle mass center & $0.279 \mathrm{~m}$ \\
\hline$a$ & distance between front axle and centroid of vehicle body & $0.894 \mathrm{~m}$ \\
\hline$b$ & distance between rear axle and centroid of vehicle body & $1.446 \mathrm{~m}$ \\
\hline$k_{s}$ & stiffness of chair & $51255 \mathrm{~N} / \mathrm{m}$ \\
\hline$k_{1}$ & tire stiffness & $96000 \mathrm{~N} / \mathrm{m}$ \\
\hline$k_{2}$ & tire stiffness & $96000 \mathrm{~N} / \mathrm{m}$ \\
\hline$k_{3}$ & stiffness of front suspension & $16500 \mathrm{~N} / \mathrm{m}$ \\
\hline$k_{4}$ & stiffness of rear suspension & $14500 \mathrm{~N} / \mathrm{m}$ \\
\hline
\end{tabular}

4 
Table 3

3 Results of the vehicle vibration analysis without consideration of mutual-correlation of the

4 road excitations

\begin{tabular}{ccc}
\hline response & maximum response interval & steady-state response interval \\
\hline$z_{\mathrm{s}}(t)$ & {$[-0.084 \mathrm{~m}, 0.084 \mathrm{~m}]$} & {$[-0.050 \mathrm{~m}, 0.050 \mathrm{~m}]$} \\
$z_{3}(t)$ & {$[-0.080 \mathrm{~m}, 0.080 \mathrm{~m}]$} & {$[-0.050 \mathrm{~m}, 0.050 \mathrm{~m}]$} \\
$\theta(t)$ & {$\left[-3.65^{\circ}, 3.65^{\circ}\right]$} & {$\left[-2.45^{\circ}, 2.45^{\circ}\right]$} \\
$z_{1}(t)$ & {$[-0.066 \mathrm{~m}, 0.066 \mathrm{~m}]$} & {$[-0.050 \mathrm{~m}, 0.050 \mathrm{~m}]$} \\
$z_{2}(t)$ & {$[-0.067 \mathrm{~m}, 0.067 \mathrm{~m}]$} & {$[-0.050 \mathrm{~m}, 0.050 \mathrm{~m}]$} \\
\hline
\end{tabular}

5

6 
Table 4

3 Results of the vehicle vibration analysis with consideration of mutual-correlation of the road

4 excitations

\begin{tabular}{ccc}
\hline response & maximum response interval & steady-state response interval \\
\hline$z_{\mathrm{s}}(t)$ & {$[-0.084 \mathrm{~m}, 0.084 \mathrm{~m}]$} & {$[-0.050 \mathrm{~m}, 0.050 \mathrm{~m}]$} \\
$z_{3}(t)$ & {$[-0.080 \mathrm{~m}, 0.080 \mathrm{~m}]$} & {$[-0.050 \mathrm{~m}, 0.050 \mathrm{~m}]$} \\
$\theta(t)$ & {$\left[-0.59^{\circ}, 0.59^{\circ}\right]$} & {$\left[-0.01^{\circ}, 0.01^{\circ}\right]$} \\
$z_{1}(t)$ & {$[-0.066 \mathrm{~m}, 0.066 \mathrm{~m}]$} & {$[-0.050 \mathrm{~m}, 0.050 \mathrm{~m}]$} \\
$z_{2}(t)$ & {$[-0.066 \mathrm{~m}, 0.066 \mathrm{~m}]$} & {$[-0.050 \mathrm{~m}, 0.050 \mathrm{~m}]$} \\
\hline
\end{tabular}

5

6 